\title{
COLLOCATING CONVOLUTIONS
}

\author{
FRANK STENGER
}

\begin{abstract}
An explicit method is derived for collocating either of the convolution integrals $p(x)=\int_{a}^{x} f(x-t) g(t) d t$ or $q(x)=\int_{x}^{b} f(t-x) g(t) d t$, where $x \in(a, b)$, a subinterval of $\mathbb{R}$. The collocation formulas take the form $\mathbf{p}=F\left(A_{m}\right) \mathbf{g}$ or $\mathbf{q}=F\left(B_{m}\right) \mathbf{g}$, where $\mathbf{g}$ is an $m$-vector of values of the function $g$ evaluated at the "Sinc points", $A_{m}$ and $B_{m}$ are explicitly described square matrices of order $m$, and $F(s)=\int_{0}^{c} \exp [-t / s] f(t) d t$, for arbitrary $c \in[(b-a), \infty]$. The components of the resulting vectors p (resp., q) approximate the values of $p$ (resp., $q$ ) at the Sinc points, and may then be used in a Sinc interpolation formula to approximate $p$ and $q$ at arbitrary points on $(a, b)$. The procedure offers a new method of approximating the solutions to (definite or indefinite) convolution-type integrals or integral equations as well as solutions of partial differential equations that are expressed in terms of convolution-type integrals or integral equations via the use of Green's functions. If $u$ is the solution of a partial differential equation expressed as a $\nu$-dimensional convolution integral over a rectangular region $\mathbf{B}$, and if $u$ is analytic and of class $\operatorname{Lip}_{\alpha}$ on the interior of each line segment in $\mathbf{B}$, then the complexity of computing an $\varepsilon$-approximation of $u$ by the method of this paper is $\mathscr{O}\left([\log (\varepsilon)]^{2 \nu+2}\right)$.
\end{abstract}

\section{INTRODUCTION AND SUMMARY}

This paper describes a new procedure for collocating an indefinite integral of convolution type. Examples are then given, illustrating the application of the procedure to the approximate solution of (indefinite or definite) convolutiontype integral equations, and to the approximate solution of (ordinary or partial) differential equations, whenever their exact solutions can be expressed via the use of Green's functions, either explicitly as an integral of convolution type, or in the form of a linear or nonlinear convolution-type integral equation.

There exist relatively few types of procedures for obtaining approximate solutions of ordinary or partial differential equations, or of integral equations. While these methods are referred to by a variety of names, such as Rayleigh's method, Ritz's method, Galerkin's method, the Bubnov-Galerkin method, the Petrov-Galerkin method, the finite element method, the spectral method, or the collocation method, they are all, in effect, variants of the same method. One first

Received by the editor October 7, 1992 and, in revised form, May 3, 1993 and September 10, 1993.

1991 Mathematics Subject Classification. Primary 65D30; Secondary 41A35, 65N35, 65R10, $65 \mathrm{~L} 10$.

Key words and phrases. Indefinite integral convolution.

Work supported by IBM grant: "Distributed Computation with Clusters of Work Stations". 
selects a suitable basis, $\left\{\omega_{j}\right\}_{j=1}^{n}$, and then attempts an approximate solution of the form

$$
\sum_{j=1}^{n} a_{j} \omega_{j}
$$

in which the coefficients $a_{j}$ are unknown. The form (1.1) is substituted into the differential or integral equation to be solved, and the coefficients $a_{j}$ are then determined in one of a variety of ways, depending on which of the above procedures is used.

The method of this paper also depends, in a sense, on a certain type of basis; nevertheless, it is quite different from any of the above-named methods. This method is based on an accurate approximation of either one or both of the convolution integrals

$$
\begin{array}{ll}
p(x)=\int_{a}^{x} f(x-t) g(t) d t, & x \in(a, b), \\
q(x)=\int_{x}^{b} f(t-x) g(t) d t, & x \in(a, b),
\end{array}
$$

where $(a, b)$ is either all of the real line $\mathbb{R}$, or else a subinterval of $\mathbb{R}$. Evidently, effective methods of approximating $p$ and $q$ in (1.2) can then be used to yield an accurate approximation to the definite convolution integral

$$
p(x)+q(x)=\int_{a}^{b} f(|x-t|) g(t) d t
$$

The approximation of Volterra-type integrals or of integral equations has been attempted by relatively few other authors. Excellent expositions of such methods may be found in Linz [7] and in Brunner [2]. By way of a quick overview, we mention the work of Young [18] who in 1954 studied the approximation of integrals of the form

$$
P(x)=\int_{0}^{x} \frac{K(x, t)}{(x-t)^{\alpha}} g(t) d t, \quad x \in(0, b),
$$

with $g$ continuous on $(0, b)$, and with $K$ continuous on $(0, b) \times(0, b)$. He discretized the integral with respect to $x$, and he then approximated $K\left(x_{i}, t\right) g(t)$ on each interval $\left[x_{i-1}, x_{i}\right]$ by a linear spline, which enabled him to carry out the resulting integrations explicitly. This procedure has been extended to more general spline approximate methods by Brunner (see, e.g., [1]), to the use of more accurate differential equation techniques by De Hoog and Weiss [3], and to the use of Sinc quadrature and Sinc collocation by Riley [12] and by Stromberg $[15,16]$, who were able to achieve arbitrary accuracy in their approximation of, e.g., $p(x)$ in (1.2), even though $f$ or $g$ (or both) could have singularities at endpoints of their respective intervals of definition. In [6] Linz recommended the use of simple quadratures, such as the trapezoidal and midordinate rules, to approximate $P(x)$ as given in (1.4). Lubich [8,9] appears to be the first to have used the Laplace transform of $f$ to approximate the integral $p(x)$ as given in (1.2). He first converted (1.2) into 


$$
\begin{aligned}
p(x) & =\int_{0}^{x} f(x-t) g(t) d t \\
& =\frac{1}{2 \pi i} \int_{\mathscr{C}} \int_{0}^{t} \hat{f}(s) \int_{0}^{x} e^{s t} g(x-t) d t d s,
\end{aligned}
$$

for which the inner integral solves the initial value problem $y^{\prime}=s y+g$, $g(0)=0$, and he then showed that the $n$th coefficient of the expansion of $\hat{f}(\delta(\zeta) / h) g(\zeta)$ in powers of $\zeta$ approximates the value $p(n h)$, where $\delta(\zeta)=$ $\left[\alpha_{0} \zeta^{k}+\cdots+\alpha_{k}\right] /\left[\beta_{0} \zeta^{k}+\cdots+\beta_{k}\right]$, and with $\alpha_{j}$ and $\beta_{j}$ denoting the usual multistep method coefficients. This procedure, which gives accurate results when one of the functions $f$ or $g$ has a singularity at an endpoint of $(0, b)$, was extended to the application of Runge-Kutta methods methods in [10].

The method of the present paper provides both formulas of high accuracy, and it uses values of $g$ at a fixed set of points on $(a, b)$, to yield an approximation of either $p$ or $q$ over the entire (finite, or semi-infinite) interval $(a, b)$. It allows for $f(x)$ to have a singularity at $x=0$, and simultaneously, for $g$ to have singularities at both endpoints of $(a, b)$. The present method thus also provides an improvement over the Fast Fourier Transform method of approximating convolutions of the form (1.3) in the case of finite or semi-infinite intervals, in the case when $f$ has a singularity at the origin, or in the case when the convolution ( $p$ or $q$ ) of $f$ and $g$ has singularities at one or both of the endpoints of $(a, b)$.

Whereas the solution to an ordinary or partial differential equation can frequently be expressed as a convolution integral of a Green's function and a known function, because of the relative lack of existence of methods of obtaining accurate approximations of (indefinite and definite) convolution-type integrals, such transformations have to date been used more often to study properties of the solution than to obtain accurate approximations of the solution. After a differential equation is transformed into such a convolution form, the method of this paper then provides an explicit procedure for obtaining an approximate solution to the differential equation. More generally, if the (linear or nonlinear) differential equation is transformed into a convolution-type integral equation via the use of Green's functions, the method of this paper enables us to replace the resulting integral equation by a system of (linear or nonlinear) algebraic equations, whose solution yields an accurate approximate solution to the differential equation.

In $\S 2$, which follows, we briefly describe the collocation procedure, and in $\S 3$ we derive the approximation scheme. In $\S 4$ we give a proof of convergence, and in $\S 5$ we illustrate a number of applications of the method, to the inversion of Laplace transforms, to the solution of Abel-type integral equations, to the solution of feedback control problems, and to the approximation of multidimensional convolution integrals. This last technique provides a new discrete separation of variables algorithm for the approximation of multidimensional convolutions, and, a fortiori, it provides a new parallel computation technique for solving any partial differential equation-elliptic, parabolic, or hyperbolic-whose solution can be written either as a multidimensional (definite or indefinite) convolution integral, or as a multidimensional (definite or indefinite) convolution-type integral equation. 


\section{APPROXIMATION PROCEDURE}

This section describes the procedure of this paper, for collocating the convolution integrals (1.2) and for obtaining explicit approximations of the functions $p$ and $q$ defined in (1.2).

Let $\mathbb{Z}$ denote the set of all integers, and let $\mathbb{C}$ denote the complex plane. Let $\operatorname{sinc}(x)$ and $e_{k}$ be defined by

$$
\begin{aligned}
\operatorname{sinc}(x) & =\frac{\sin (\pi x)}{\pi x}, \\
\sigma_{k} & =\int_{0}^{k} \operatorname{sinc}(x) d x, \\
e_{k} & =\frac{1}{2}+\sigma_{k}, \quad k \in \mathbf{Z} .
\end{aligned}
$$

Note that $\sigma_{-k}=-\sigma_{k}$, and we therefore only need to compute $\sigma_{k}$ for positive integers $k$. One way of computing the $\sigma_{k}$ is via the recurrence $\sigma_{k+1}=\sigma_{k}+$ $(-1)^{k} \nu_{k}$, where $\nu_{k}=\int_{0}^{1}\{\sin (\pi x)\} d x /\{\pi(x+k)\}$, and where the $\nu_{k}$ can be accurately and efficiently computed via Gauss-Legendre quadrature.

Let $\phi$ denote a one-to-one transformation of the interval $(a, b)$ onto the real line $\mathbb{R}$, let $h$ denote a fixed positive number, and let the Sinc points be defined on $(a, b)$ by $z_{k}=\phi^{-1}(k h), k \in \mathbf{Z}$, where $\phi^{-1}$ denotes the inverse function of the function $\phi$. Let $M$ and $N$ be positive integers, set $m=M+N+1$, and for a given function $u$ defined on $(a, b)$, define a diagonal matrix $D(u)$ by $D(u)=\operatorname{diag}\left[u\left(z_{-M}\right), \ldots, u\left(z_{N}\right)\right]$. Let $I^{(-1)}$ be a square matrix of order $m$ having $e_{i-j}$ as its $(i, j)$ th element, $i, j=-M, \ldots, N$. Define square matrices $A_{m}$ and $B_{m}$ by

$$
A_{m}=h I^{(-1)} D\left(1 / \phi^{\prime}\right), \quad B_{m}=h\left\{I^{(-1)}\right\}^{T} D\left(1 / \phi^{\prime}\right),
$$

where the superscript " $T$ " denotes the transpose. Throughout the paper, when referring to the Laplace transform of a function $f$ we will mean the usual Laplace transform, $\hat{f}(s)=\int_{0}^{c} e^{-s t} f(t) d t$, whereas when referring to the Laplace transform in quotations, i.e., the "Laplace transform", will mean the function $F$ defined by $F(s)=\hat{f}(1 / s)$, i.e.,

$$
F(s)=\int_{0}^{c} e^{-t / s} f(t) d t
$$

We shall assume that the "Laplace transform" exists for some $c \in[b-a, \infty]$, for all $s$ on the right half of the complex plane $\Omega^{+}=\{z \in \mathbb{C}: \mathfrak{R} z>0\}$. Although these conditions suffice for purposes of defining the convolution approximations of this paper, in $\S 4$ of this paper we shall require more stringent conditions on the "Laplace transform" for purposes of obtaining an accurate error bound on the approximation. For example, in $\S 4$ we shall require that the "Laplace transform" should exist for some $c \in[2(b-a), \infty]$, and for all $s \in \Omega^{+}$. In applications it is most convenient to simply look up the Laplace transform $\hat{f}(s)$ in a table, whenever this is possible. The choice $c=\infty$ is fine, whenever the integral in (2.3) exists for $c=\infty$. On the other hand, it may be necessary to take $c$ to be finite. This is the case, for example, if $(a, b)=(0,1)$, and $f(t)=t^{-1 / 3} \exp \left\{t^{2}\right\}$. It is probably best to take $c$ as small as possible if $F(s)$ 
has to be approximated via numerical integration, although the approximation of $F$ can usually be done effectively via Sinc quadrature (see $[13, \S \S 4.2,6.9]$ ), regardless of the length of the interval.

The eigenvalues of each of the matrices $A_{m}$ and $B_{m}$ defined in (2.2) belong to $\overline{\Omega^{+}}$(actually it has been shown by numerical computation that these eigenvalues belong to $\Omega^{+}$for $\left.1 \leq m \leq 513\right)$. Thus, if nonsingular matrices $X_{m}, Y_{m}$, and complex numbers $s_{m, j}$ are determined such that

$$
\begin{aligned}
& A_{m}=X_{m} \operatorname{diag}\left[s_{m,-M}, \ldots, s_{m, N}\right] X_{m}^{-1}, \\
& B_{m}=Y_{m} \operatorname{diag}\left[s_{m,-M}, \ldots, s_{m, N}\right] Y_{m}^{-1},
\end{aligned}
$$

then square matrices $F\left(A_{m}\right)$ and $F\left(B_{m}\right)$ may be defined via the equations

$$
\begin{aligned}
& F\left(A_{m}\right)=X_{m} \operatorname{diag}\left[F\left(s_{m,-M}\right), \ldots, F\left(s_{m, N}\right)\right] X_{m}^{-1}, \\
& F\left(B_{m}\right)=Y_{m} \operatorname{diag}\left[F\left(s_{m,-M}\right), \ldots, F\left(s_{m, N}\right)\right] Y_{m}^{-1} .
\end{aligned}
$$

We have tacitly assumed here that $A_{m}$ and $B_{m}$ are diagonalizable (see the pertaining discussion in $\S 4$ ).

Now, define column vectors $\mathbf{g}_{m}, \mathbf{p}_{m}$, and $\mathbf{q}_{m}$ by

$$
\begin{aligned}
& \mathbf{g}_{m}=\left(g\left(z_{-M}\right), \ldots, g\left(z_{N}\right)\right)^{T}, \\
& \mathbf{p}_{m}=\left(p_{-M}, \ldots, p_{N}\right)^{T}=F\left(A_{m}\right) \mathbf{g}_{m}, \\
& \mathbf{q}_{m}=\left(q_{-M}, \ldots, q_{N}\right)^{T}=F\left(B_{m}\right) \mathbf{g}_{m} .
\end{aligned}
$$

The component $p_{j}$ of the vector $\mathbf{p}_{m}$ (resp., $q_{j}$ of the vector $\mathbf{q}_{m}$ ) approximates the value $p(x)$ (resp., $q(x)$ ) at the Sinc point $x=z_{j}$.

The next step of the procedure is to describe a basis, $\left\{\omega_{j}\right\}_{j=-M}^{N}$, such that the vectors $\mathbf{p}_{m}$ and $\mathbf{q}_{m}$ determined as in (2.6) may be used to approximate the functions $p(x)$ and $q(x)$ throughout $(a, b)$, where $p(x)$ and $q(x)$ were defined as in (1.2). Letting $\operatorname{sinc}(x)$ be defined as in (2.1), we set

$$
\begin{aligned}
\gamma_{j}(x) & =\operatorname{sinc}\{[\phi(x)-j h] / h\}, \quad j=-M, \ldots, N, \\
\omega_{j}(x) & =\gamma_{j}(x), \quad j=-M+1, \ldots, N-1, \\
\omega_{-M}(x) & =\left[1+e^{-M h}\right]\left[\frac{1}{1+\rho(x)}-\sum_{j=-M+1}^{N} \frac{\gamma_{j}(x)}{1+e^{j h}}\right], \\
\omega_{N}(x) & =\left[1+e^{-N h}\right]\left[\frac{\rho(x)}{1+\rho(x)}-\sum_{j=-M}^{N-1} \frac{e^{j h} \gamma_{j}(x)}{1+e^{j h}}\right],
\end{aligned}
$$

with $\rho=e^{\phi}$. The functions $\left\{\omega_{j}\right\}_{j=-M}^{N}$ defined in (2.7) satisfy the relations $\omega_{j}\left(z_{k}\right)=\delta_{j, k}$, where $\delta_{j, k}$ denotes the Kronecker delta. We remark that the multiplicative factors $\left[1+e^{-M h}\right]$ and $\left[1+e^{-N h}\right]$ (which enable us to write $\left.\omega_{j}\left(z_{k}\right)=\delta_{j, k}\right)$ can in fact be dropped for purposes of carrying out interpolation in practice, since, e.g., the exclusion of these factors does not alter the statement of Theorem 4.2 below.

The approximations of $p$ and $q$ on $(a, b)$ then take the form

$$
p(x) \approx \sum_{j=-M}^{N} p_{j} \omega_{j}(x), \quad q(x) \approx \sum_{j=-M}^{N} q_{j} \omega_{j}(x) .
$$


The basis functions defined in (2.7) suffice for purposes of uniform-norm approximation over $(a, b)$. They are also convenient for purposes of uniformnorm approximation in that the same basis functions can then be used to approximate both functions $p$ and $q$, and hence also the sum, $p+q$. On the other hand, it may at times be convenient to replace $\omega_{-M}(x)$ by $\gamma_{-M}(x)$, or $\omega_{N}(x)$ by $\gamma_{N}(x)$, with $\gamma_{j}(x)$ defined in the first line of (2.7), if it is desirable that the final approximation vanish at an endpoint of the interval, since the functions $\gamma_{j}(x)$ vanish at both endpoints of the interval of approximation. For example, for purposes of approximating $p$ on $(a, b)$ via the formula in (2.8), an equally accurate uniform-norm approximation obtains, if instead of the basis in (2.7), we select the basis

$$
\begin{aligned}
\omega_{j}(x) & =\operatorname{sinc}\{[\phi(x)-j h] / h\}, \quad j=-M, \ldots, N-1, \\
\omega_{N}(x) & =\left[1+e^{-N h}\right]\left[\frac{\rho(x)}{1+\rho(x)}-\sum_{j=-M}^{N-1} \frac{e^{j h} \omega_{j}(x)}{1+e^{j h}}\right] .
\end{aligned}
$$

Furthermore, by using (2.9), the resulting approximation as defined in (2.8) then vanishes at the endpoint $a$. An obviously similar modification of (2.7) is possible for purposes of getting an approximation of $q$, which vanishes at $b$.

More specific descriptions will be given below for the selections of $\phi, h, M$, and $N$, as well as for a space of functions of the type that one usually encounters in applications, and these more explicit descriptions will then make it possible to obtain accurate bounds on the error of the approximations (2.8).

\section{Formula DERIVATION}

It suffices to consider only the case of $p(x)$ as defined in (1.2), since the case of $q(x)$ can be treated in exactly the same way. Let $\mathscr{I} w$ be defined for $w \in \mathbf{L}^{1}(a, b)$ by

$$
(\mathscr{I} w)(x)=\int_{a}^{x} w(t) d t .
$$

It then follows that, for $n=1,2, \ldots$,

$$
\left(\mathscr{I}^{n} w\right)(x)=\int_{a}^{x} \frac{(x-t)^{n-1}}{(n-1) !} w(t) d t
$$

The length of the interval $(a, b)$ will at the outset be assumed to be finite. It is then convenient to take $\|w\|=(b-a)^{-1} \int_{a}^{b}|w(t)| d t$, since this choice of norm yields the simple inequality

$$
\|\mathscr{F} w\|=\frac{1}{b-a} \int_{a}^{b}\left|\int_{a}^{x} w(t) d t\right| d x \leq(b-a)\|w\|,
$$

which, together with (3.2), implies that

$$
\left\|\mathscr{I}^{n}\right\| \leq \frac{(b-a)^{n}}{n !}, \quad\left\|\mathscr{I}^{n}\right\| \leq\|\mathscr{I}\|^{n} \leq(b-a)^{n} .
$$

By using the Bromwich formula $[17$, p. 67] for the inversion of the Laplace transform, and then converting to the "Laplace transform" via replacement of 
$s$ by $1 / s$ (a transformation which transforms $\Omega^{+}$to itself) the expression (1.2) for $p(x)$ may be written in the alternate forms

$$
\begin{aligned}
p(x) & =-\int_{a}^{x} \frac{1}{2 \pi i} \int_{-i \infty}^{i \infty} e^{(x-t) / s} F(s) s^{-2} d s g(t) d t \\
& =-\frac{1}{2 \pi i} \int_{-i \infty}^{i \infty} w(x, s) d s
\end{aligned}
$$

where $F(s)$ is given by (2.3), and where

$$
w(x, s)=\int_{a}^{x} s^{-2} e^{(x-t) / s} F(s) g(t) d t
$$

Now, if $|s|>b-a$, and we use the notations of (3.1) and (3.2), as well as the inequality (3.3), we get

$$
\begin{aligned}
w(x, s) & =\int_{a}^{x} \sum_{n=0}^{\infty} \frac{(x-t)^{n}}{n ! s^{n+2}} F(s) g(t) d t \\
& =\left(\frac{\mathscr{I}}{s^{2}} \sum_{n=0}^{\infty} \frac{\mathscr{I}^{n}}{s^{n}} F(s) g\right)(x)=\left(\frac{\mathscr{I}}{s}(s-\mathscr{I})^{-1} F(s) g\right)(x) .
\end{aligned}
$$

By analytic continuation as a function of $s$, it then follows that the identity

$$
\int_{a}^{x} s^{-2} e^{(x-t) / s} F(s) g(t) d t=\left(\frac{\mathscr{I}}{s}(s-\mathscr{I})^{-1} F(s) g\right)(x)
$$

holds not only for all $s \in \mathbb{C}$ such that $|s|>|b-a|$, but in the larger, resolvent set of $\mathcal{I}$, excluding the point $s=0$. Here, the resolvent set of $\mathscr{I}$ is the set $\left\{s \in \mathbb{C}:(s-\mathscr{I})^{-1}\right.$ exists $\}$. The resolvent set of $\mathscr{I}$ can be more closely identified, upon setting

$$
(u, v) \equiv \int_{a}^{b} u(x) \overline{v(x)} d x
$$

It follows, in this notation, that

$$
\mathfrak{R}(\mathscr{I} u, u)=\frac{1}{2}\left|\int_{a}^{b} u(x) d x\right|^{2} \geq 0
$$

Hence, the resolvent set of $\mathscr{I}$ includes the set $\{s \in \mathbb{C}: \mathfrak{R} s<0\}$, as well as the set $\{s \in \mathbb{C}:|s|>b-a\}$.

Substitution of (3.8) into (3.5) yields the Dunford-type integral

$$
p(x)=-\lim _{\varepsilon \rightarrow 0^{+}}\left(\frac{1}{2 \pi i} \int_{-i \infty}^{i \infty} \frac{\mathscr{I}}{s-\varepsilon}(s-\varepsilon-\mathscr{I})^{-1} F(s) d s g\right)(x),
$$

where we have defined this integral as a limit $\lim _{\varepsilon \rightarrow 0}$, since we have only established above that the spectrum of $\mathscr{I}$ lies in the closed (and not the open) right half-plane. If $(a, b)$ is a finite interval, then it follows that $F(s)$ is bounded for all sufficiently large $s \in \mathbb{C}$. We may thus use Cauchy's formula in (3.11), to evaluate the integral by summing the residues at the "poles", at $s=\mathscr{I}+\varepsilon$ and at $s=\varepsilon$, and then let $\varepsilon \rightarrow 0$ in the result. Since the classical Laplace transform 
$\hat{f}(s) \equiv \int_{0}^{c} e^{-s t} f(t) d t \rightarrow 0$ as $s \rightarrow \infty$, it follows that $F(0)=\hat{f}(\infty)=0$. Hence (3.11) is equivalent to the expression

$$
p(x)=-\left(\frac{1}{2 \pi i} \int_{-i \infty}^{i \infty} \frac{\mathscr{I}}{s}(s-\mathscr{I})^{-1} F(s) d s g\right)(x) .
$$

Either (3.11) or (3.12) thus yields the formula

$$
p(x)=(F(\mathscr{I}) g)(x),
$$

which is valid for bounded intervals $(a, b)$.

One way of interpreting the expression $F(\mathscr{I}) g$ is the following: Let $\left\{\mathscr{B}_{j}\right\}_{j=1}^{\infty}$ denote a sequence of simply connected open domains each having compact closure, and each lying in $\Omega^{+}$, such that $\mathscr{B}_{j} \subset \mathscr{B}_{j+1}$, and such that $\lim _{j \rightarrow \infty} \mathscr{B}_{j}=$ $\Omega^{+}$. For each fixed $j$, let $\left\{p_{j, m}(s)\right\}_{m=0}^{\infty}$ denote a sequence of polynomials, with $p_{j, m}(s)$ of degree $m$ in $s$, such that $p_{j, m}(s)$ converges uniformly to $F(s)$ in the closure of $\mathscr{B}_{j}$. Then $p_{n, n}(s) \rightarrow F(s)$ for all $s \in \Omega^{+}$. The operator $\left(p_{n, n}(\mathscr{J}) g\right)(x)$ is of course well defined for every positive integer $n$, and we can define $(F(\mathscr{I}) g)(x)$ by $(F(\mathscr{I}) g)(x)=\lim _{n \rightarrow \infty}\left(p_{n, n}(\mathscr{I}) g\right)(x)$.

Another (equivalent) way of interpreting the expression $F(\mathscr{I}) g$ is to note from above that

$$
\begin{aligned}
w(x, s) & \equiv\left(\frac{\mathscr{I}}{s}[s-\mathscr{I}]^{-1} F(s) g\right)(x) \\
& =\frac{1}{s^{2}} \int_{a}^{x} e^{(x-t) / s} F(s) g(t) d t
\end{aligned}
$$

Hence, recalling the above definition of $\mathscr{B}_{j}$, it thus follows from the Dunford integral expression (3.12) that

$$
(F(\mathscr{I}) g)(x)=\lim _{j \rightarrow \infty} \frac{-1}{2 \pi i} \int_{\partial \mathscr{B}_{j}} w(x, s) d s .
$$

Recall now the definition (2.3) of $F$, which implies that $F=F_{1}+F_{2}$, where $F_{1}(s)=\int_{0}^{b-a} e^{-t / s} f(t) d t$, and where $F_{2}(s)=\int_{b-a}^{c} e^{-t / s} f(t) d t$. It follows from this, and the Bromwich inversion formula, that if $t<b-a$, then $(2 \pi i)^{-1} \int_{-i \infty}^{i \infty} e^{t / s} F_{2}(s) s^{-2} d s=0$. The formula (3.12) still holds in the case when $c>b-a$, since in that case, in view of the above remarks, it follows, upon recalling that $g(t)=0$ for $t>b-a$, that $F_{2}(\mathscr{I}) g=0$, and therefore $F(\mathscr{J}) g=F_{1}(\mathscr{I}) g$.

Next, assume that $(a, b)$ is an unbounded interval, and assume for this case that the function $p$ defined in (1.2) exists for fixed $f$ and a dense subspace $\mathbf{X}$ of functions $g \in \mathbf{L}^{1}(a, b)$ such as the space $\mathbf{C}[a, b]$ of continuous functions on $[a, b]$. The space $\mathbf{X}$ will be defined more precisely in $\S 4$ below. The expression (3.13) can then again be established to be valid for all $g \in \mathbf{X}$, by repeating the above argument for each of a sequence of nested finite intervals $\left(a_{j}, b_{j}\right) \subset$ $\left(a_{j+1}, b_{j+1}\right)$, where $a_{j} \rightarrow a$ and $b_{j} \rightarrow b$ as $j \rightarrow \infty$. We furthermore need to assume that $F(s) / s^{2}=o(1 / s)$ as $s \rightarrow \infty$ in $\Omega^{+}$, in order to be able to apply the residue theorem to get (3.13) from (3.11) or from (3.12). On the other hand, if $F(s) \sim \gamma s$ as $s \rightarrow \infty$, then $F(s)-\gamma s=o(s)$ as $s \rightarrow \infty$, i.e., we can in fact assume that (3.12) holds even if $F(s)=\mathscr{O}(s)$ as $s \rightarrow \infty$.

Now define linear operators $V_{m}, \Pi_{m}$, and $\mathscr{I}_{m}$ as follows. For $g$ defined and continuous on $(a, b)$, set $V_{m} g=\left(g\left(z_{-M}\right), \ldots, g\left(z_{N}\right)\right)^{T}$, and for a given vector $\mathbf{c}=\left(c_{-M}, \ldots, c_{N}\right)^{T}$, define $\Pi_{m} \mathbf{c}$ by $\left(\Pi_{m} \mathbf{c}\right)(x)=\sum_{j=-M}^{N} c_{j} \omega_{j}(x)$, where 
the basis functions $\omega_{j}$ are defined as in (2.7). Then, define $\mathscr{I}_{m}$ on $\mathbf{X}$ by $\mathscr{J}_{m}=\Pi_{m} A_{m} V_{m}$, where the matrix $A_{m}$ is defined as in (2.2). That is,

$$
\begin{aligned}
V_{m} g & =\left(g\left(z_{-M}\right), \ldots, g\left(z_{N}\right)\right)^{T}, \\
\mathbf{c} & =\left(c_{-M}, \ldots, c_{N}\right)^{T}, \\
\left(\Pi_{m} \mathbf{c}\right)(x) & =\sum_{j=-M}^{N} c_{j} \omega_{j}(x), \\
\left(\mathscr{I}_{m} g\right)(x) & =\left(\Pi_{m} A_{m} V_{m} g\right)(x) .
\end{aligned}
$$

Since $V_{m} \Pi_{m}$ is then the unit matrix of order $m$, it follows, upon recalling the above definition (2.5) of $F\left(A_{m}\right)$, that $F\left(\mathscr{J}_{m}\right)$ is given by

$$
F\left(\mathscr{I}_{m}\right)=\Pi_{m} F\left(A_{m}\right) V_{m} \text {. }
$$

The operator $F\left(\mathscr{I}_{m}\right)$ could, of course, also be defined in the same way that the operator $F(\mathscr{F})$ was defined above, either as a Dunford operator-valued integral, or as a limit of a sequence of polynomials in the variable $\mathscr{I}_{m}$, although these latter definitions have little practical value. On the other hand, the expression (3.17) yields a practically useful definition of $F\left(\mathscr{J}_{m}\right)$ and moreover, it provides a practically useful method of approximating $F(\mathscr{I}) g$. The expression (3.13) can thus be replaced by the approximating one

$$
p(x)=(F(\mathscr{I}) g)(x) \approx\left(F\left(\mathscr{I}_{m}\right) g\right)(x)=\left(\Pi_{m} F\left(A_{m}\right) V_{m} g\right)(x) .
$$

The convergence and error of this approximation are discussed in the following section.

\section{Convergence}

The procedure of the previous section has already been tested on a variety of partial differential equation and convolution integral problems. Since the procedure is based on Sinc approximations, the proof of convergence given below is for spaces of functions in which Sinc approximation is close to optimal. Only the case of $\int_{a}^{x} f(x-t) g(t) d t$ is considered throughout this section, since the considerations for $\int_{x}^{b} f(t-x) g(t) d t$ are essentially the same.

Definition 4.1. Let $d \in(0, \pi)$, and let $\mathscr{D}_{d}=\{z \in \mathbb{C}:|\mathfrak{I} z|<d\}$. Let the interval $(a, b)$ be defined as in the previous section, and let $\phi$ be a conformal map of a simply connected domain $\mathscr{D}$ onto $\mathscr{D}_{d}$, such that $\mathscr{D}$ contains the interval $(a, b)$, and such that $\phi((a, b))=\mathbb{R}$. Clearly, if $d^{\prime}$ is a number such that $d^{\prime}>d$, and if the same function $\phi$ provides a conformal map of $\mathscr{D}^{\prime}$ onto $\mathscr{D}_{d^{\prime}}$, then $\mathscr{D} \subset \mathscr{D}^{\prime}$. Set $\rho=e^{\phi}$. Let $\alpha$ and $\beta$ denote positive numbers, and let $\mathbf{L}_{\alpha, \beta}(\mathscr{D})$ denote the family of all functions $f \in \operatorname{Hol}(\mathscr{D})$, for which there exists a positive constant $c_{1}$ such that, for all $z \in \mathscr{D}$,

$$
|f(z)| \leq c_{1} \frac{|\rho(z)|^{\alpha}}{[1+|\rho(z)|]^{\alpha+\beta}} .
$$

Now let the positive numbers $\alpha$ and $\beta$ belong to $(0,1]$, and let $\mathbf{M}_{\alpha, \beta}(\mathscr{D})$ denote the family of all functions $g \in \operatorname{Hol}(\mathscr{D})$, such that $g(a)$ and $g(b)$ are finite numbers, where $g(a)=\lim _{z \rightarrow a} g(z)$ and $g(b)=\lim _{z \rightarrow b} g(z)$, and such that $f \in \mathbf{L}_{\alpha, \beta}(\mathscr{D})$, where

$$
f(z)=g(z)-\frac{g(a)+\rho(z) g(b)}{1+\rho(z)} .
$$


We remark that the spaces $\mathbf{L}_{\alpha, \beta}(\mathscr{D})$ and $\mathbf{M}_{\alpha, \beta}(\mathscr{D})$ are invariant under conformal transformation, in the sense that if $\phi_{j}$ denotes a conformal map of $\mathscr{D}_{j}$ onto $\mathscr{D}_{d}$, for $j=1,2$, and if, e.g., $u \in \mathbf{L}_{\alpha, \beta}\left(\mathscr{D}_{1}\right)$, then $v=u \circ \phi_{1}^{-1} \circ \phi_{2} \in \mathbf{L}_{\alpha, \beta}\left(\mathscr{D}_{2}\right)$.

The space of functions $g \in \operatorname{Hol}(\mathscr{D})$ that are absolutely integrable over the boundary of $\mathscr{D}$ will be denoted by $\mathbf{X}$. The space of all functions $w \in$ $\operatorname{Hol}(\mathscr{D})$ that are uniformly bounded in $\mathscr{D}$ will be denoted by $\mathbf{Y}$. The spaces $\mathbf{X}$ and $\mathbf{Y}$ may be normed by $\|g\|_{\mathbf{X}}=\sup _{x \in(a, b)}\left|g(x) / \phi^{\prime}(x)\right|$ and $\|w\|_{\mathbf{Y}}=$ $\sup _{x \in(a, b)}|w(x)|$, respectively.

Corresponding to a positive integer $N$, determine $h$ via the formula $h=$ $c / N^{1 / 2}$, where $c$ is constant, take $M=[\beta / \alpha N]$, where $[x]$ denotes the greatest integer in $x$, and then set $m=M+N+1$. The Sinc points $z_{j}$ are determined by the formula $z_{j}=\phi^{-1}(j h)$. Given $g \in \mathbf{X}$, let $\mathscr{I} g$ and $\mathscr{I}_{m} g$ be defined as in (3.1) and (3.16), respectively. It may be shown, for example (see [13]), that if $g / \phi^{\prime} \in \mathbf{L}_{\alpha^{\prime}, \beta^{\prime}}(\mathscr{D})$ (resp., $g \in \mathbf{X}$ ), then $\mathscr{I} g \in \mathbf{M}_{\alpha, \beta}(\mathscr{D}), \alpha=\min \left(1, \alpha^{\prime}\right)$, and $\beta=\min \left(1, \beta^{\prime}\right)$ (resp., $\left.\mathscr{I} g \in \mathbf{Y}\right)$. On the other hand, if $u \in \mathbf{M}_{\alpha, \beta}\left(\mathscr{D}^{\prime}\right)$ (resp., $u \in \mathbf{Y}\left(\mathscr{D}^{\prime}\right)$ ), then $u^{\prime} / \phi^{\prime} \in \mathbf{L}_{\alpha, \beta}(\mathscr{D})$ (resp., $u^{\prime} / \phi^{\prime} \in \mathbf{Y}(\mathscr{D})$ ). Since $0<d<\pi$, and since $\alpha \in(0,1]$, and $\beta \in(0,1]$, it follows that $\mathbf{M}_{\alpha, \beta}(\mathscr{D}) \subset \mathbf{Y}$.

For purposes of approximating in the spaces $\mathbf{Y}$ and $\mathbf{M}_{\alpha, \beta}(\mathscr{D})$ we record Theorem $4.2([13,14])$. (a) Let $u \in \mathbf{Y}$, let $\omega_{j}$ be defined as in (2.7), and let $M$ and $N$ be defined as in Definition 4.1. If $h=\gamma / N^{1 / 2}$, where $\gamma$ is a positive constant, then as $N \rightarrow \infty$,

$$
\left\|u-\sum_{j=-M}^{N} u\left(z_{j}\right) \omega_{j}\right\|_{\mathbf{Y}} \rightarrow 0 .
$$

In particular, if $u \in \mathbf{M}_{\alpha, \beta}(\mathscr{D})$, and if $h=[\pi d /(\beta N)]^{1 / 2}$, then there exists a positive constant $c_{1}$ which is independent of $N$, such that

$$
\left\|u-\sum_{j=-M}^{N} u\left(z_{j}\right) \omega_{j}\right\|_{\mathbf{Y}} \leq c_{1} N^{1 / 2} e^{-(\pi d \beta N)^{1 / 2}} .
$$

The choice $h=[\pi d /(\beta N)]^{1 / 2}$ is close to optimal for approximation in the space $\mathbf{M}_{\alpha, \beta}(\mathscr{D})$ in the sense that the error bound in (4.4) cannot be appreciably improved regardless of the basis; if instead of this choice of $h$, we select $h=\gamma / N^{1 / 2}$, then we get the error bound $c e^{-\delta N^{1 / 2}}$, in which we may take $\delta=\min (\pi d / \gamma, \beta \gamma)$.

It may be readily shown that the eigenvalues of the matrix $A_{m}$ defined in (2.2) lie in the closure of the right half-plane, $\Omega^{+}$. To this end, we mention that $I^{(-1)}=H+S$, where each of the $m^{2}$ entries of $H$ is the number $1 / 2$, and where $S$ is a square matrix of order $m$ having $\sigma_{i-j}$ as its $(i, j)$ th element. Hence, if $\mathbf{c}=\left(c_{-M}, \ldots, c_{N}\right)^{T}$ is an arbitrary complex vector of order $m$,

$$
\mathbf{c}^{*} I^{(-1)} \mathbf{c}=\frac{1}{2}\left|\sum_{j=-M}^{N} c_{j}\right|^{2}+\mathbf{c}^{*} S \mathbf{c} .
$$

Since the term $\mathrm{c}^{*} S \mathrm{c}$ is purely imaginary, it is clear that the eigenvalues of $I^{(-1)}$ all lie in the closure of the right half-plane. Indeed, it has already been shown 
by numerical computation that the matrix $I^{(-1)}$ of order $m$ is diagonalizable, and moreover, the real part of each eigenvalue of $I^{(-1)}$ is positive, for every integer $m \in[1,513]$. Whereas the attainable accuracy of Sinc approximation is problem-dependent, a value of $m=15$ will usually yield about 3 places of accuracy, a value of $m=30$ will yield about 5 , and a value of $m=513$ should yield at least 25 places of accuracy. It will thus be assumed that $m$ is such that $I^{(-1)}$ is diagonalizable, and that the real part of every eigenvalue of $I^{(-1)}$ is positive. Next, since $A_{m}=I^{(-1)} D$, where $D=D\left(1 / \phi^{\prime}\right)$, and each entry $d_{j}$ of the diagonal matrix $D$ is positive, and since the eigenvalues of $A_{m}$ are the same as the eigenvalues of $D^{1 / 2} A_{m} D^{-1 / 2}=D^{1 / 2} I^{(-1)} D^{1 / 2}$, it follows, in the notation of $(4.5)$, that

$$
\mathbf{c}^{*} D^{1 / 2} I^{(-1)} D^{1 / 2} \mathbf{c}=\frac{1}{2}\left|\sum_{j=-M}^{N} c_{j} d_{j}\right|^{2}+\mathbf{c}^{*} D^{1 / 2} S D^{1 / 2} \mathbf{c} .
$$

The term involving the sum on the right-hand side of (4.6) is nonnegative, whereas the inner-product term involving the matrix $S$ is purely imaginary, which again leads to the conclusion that the eigenvalues of $A_{m}$ lie on the closure of the right half-plane. Indeed, the above discussion shows that the real parts of the eigenvalues of $A_{m}$ are positive if and only if the real parts of the eigenvalues of $I^{(-1)}$ are positive. Unfortunately, we have not been able to show theoretically that the matrix $I^{(-1)} D\left(1 / \phi^{\prime}\right)$ is always diagonalizable, although this has been the case for all of the examples that we have attempted to date. It will therefore be assumed that $A_{m}=I^{(-1)} D$ is such that the real part of every eigenvalue of $A_{m}$ is positive, and that $A_{m}$ is diagonalizable. It follows, under this assumption, that every eigenvalue of the matrix $B_{m}=\left(I^{(-1)}\right)^{T} D$ lies on the right half-plane, and that this matrix is diagonalizable, and also, that the matrices $F\left(A_{m}\right)$ and $F\left(B_{m}\right)$ are well defined.

The examples which follow exhibit finite, semi-infinite, and infinite intervals, and corresponding functions $\phi$, which allow for either algebraic or exponential approach to limits, as either $x \rightarrow a$ or as $x \rightarrow b$.

Example 4.3. For example, if $(a, b)=(0,1)$, and if $\mathscr{D}$ is the "eye-shaped" region, $\mathscr{D}=\{z \in \mathbb{C}:|\arg [z /(1-z)]|<d\}$, then $\phi(z)=\log [z /(1-z)]$, the relation (4.2) reduces to $f=g-(1-x) g(0)-x g(1)$, and the class $\mathbf{L}_{\alpha, \beta}(\mathscr{D})$ described by (4.1) is the class of all functions $f \in \operatorname{Hol}(\mathscr{D})$, such that for all $z \in \mathscr{D},|f(z)|<c|z|^{\alpha}|1-z|^{\beta}$. In this case, if, e.g., $\delta=\max \{\alpha, \beta\}$, and a function $w$ is such that $w \in \operatorname{Hol}(\mathscr{D})$, and $w \in \operatorname{Lip}_{\delta}(\mathscr{D})$, then $w \in \mathbf{M}_{\alpha, \beta}(\mathscr{D})$. The Sinc points $z_{j}$ are $z_{j}=e^{j h} /\left(1+e^{j h}\right)$, and $1 / \phi^{\prime}\left(z_{j}\right)=e^{j h} /\left(1+e^{j h}\right)^{2}$. This transformation $\phi$ enables the approximation of convolution integrals of the type

$$
p(x)=\int_{0}^{x}(x-t)^{c^{\prime}-b^{\prime}-1} t^{b^{\prime}-1}(1-t)^{-a^{\prime}} d t .
$$

Example 4.4. If $(a, b)=(0, \infty)$, and if $\mathscr{D}$ is the "sector" $\mathscr{D}=\{z \in \mathbb{C}$ : $|\arg (z)|<d\}$, then $\phi(z)=\log (z)$, the relation (4.2) reduces to $f(z)=g(z)-$ $[g(0)+z g(\infty)] /(1+z)$, and the class $\mathbf{L}_{\alpha, \beta}(\mathscr{D})$ is the same as the class of all functions $f \in \operatorname{Hol}(\mathscr{D})$ such that, if $z \in \mathscr{D}$ and $|z| \leq 1$, then $|f(z)| \leq c|z|^{\alpha}$, while if $z \in \mathscr{D}$ and $|z| \geq 1$, then $|f(z)| \leq c|z|^{-\beta}$. This map thus allows for 
algebraic decay at both $x=0$ and $x=\infty$. The Sinc points $z_{j}$ are defined by $z_{j}=e^{j h}$, and $1 / \phi^{\prime}\left(z_{j}\right)=e^{j h}$. This transformation enables the approximation of convolution integrals such as

$$
p(x)=\int_{0}^{x}(x-t)^{a^{\prime}-1} t^{b^{\prime}-1}(1+t)^{-c^{\prime}} d t .
$$

Example 4.5. If $(a, b)=(0, \infty)$, and if $\mathscr{D}$ is the "bullet-shaped" region $\mathscr{D}=$ $\{z \in \mathbb{C}:|\arg [\sinh (z)]|<d\}$, then $\phi(z)=\log (\sinh (z))$. The relation (4.2) then reduces to

$$
f(z)=g(z)-[g(0)+\sinh (z) g(\infty)] /(1+\sinh (z)),
$$

and the class $\mathbf{L}_{\alpha, \beta}(\mathscr{D})$ is the same as the class of all functions $f \in \operatorname{Hol}(\mathscr{D})$ such that, if $z \in \mathscr{D}$ and $|z| \leq 1$, then $|f(z)| \leq c|z|^{\alpha}$, while if $z \in \mathscr{D}$ and $|z| \geq 1$, then $|f(z)| \leq c \exp \{-\beta|z|\}$. This map thus allows for algebraic decay at $x=0$ and exponential decay at $x=\infty$. The Sinc points $z_{j}$ are defined by $z_{j}=\log \left[e^{j h}+\left(1+e^{2 j h}\right)^{1 / 2}\right]$, and $1 / \phi^{\prime}\left(z_{j}\right)=\left(1+e^{-2 j h}\right)^{-1 / 2}$. This transformation enables the approximation of convolution integrals such as

$$
q(x)=\int_{x}^{\infty}(t-x)^{a^{\prime}-1} t^{b^{\prime}-1} e^{-c^{\prime} t} d t
$$

Example 4.6. If $(a, b)=\mathbb{R}$, and if $\mathscr{D}$ is the above-defined "strip", $\mathscr{D}=\mathscr{D}_{d}$, take $\phi(z)=z$. The relation (4.2) then reduces to

$$
f(z)=g(z)-\left[g(-\infty)+e^{z} g(\infty)\right] /\left(1+e^{z}\right) .
$$

The class $\mathbf{L}_{\alpha, \beta}(\mathscr{D})$ is the same as the class of all functions $f \in \operatorname{Hol}(\mathscr{D})$ such that, if $z \in \mathscr{D}$ and $\mathfrak{R} z \leq 0$, then $|f(z)| \leq c e^{-\alpha|z|}$, while if $z \in \mathscr{D}$ and $\mathfrak{R} z \geq 0$, then $|f(z)| \leq c e^{-\bar{\beta}|z|}$. Thus, this map allows for exponential decay at both $x=-\infty$ and $x=\infty$. The Sinc points $z_{j}$ are defined by $z_{j}=j h$, and $1 / \phi^{\prime}\left(z_{j}\right)=1$. This transformation enables the approximation of integrals such as

$$
p(x)=\int_{-\infty}^{x} \frac{d t}{\cosh \left[b^{\prime}(x-t)\right] \cosh \left(c^{\prime} t\right)}
$$

Example 4.7. If $(a, b)=\mathbb{R}$, and if $\mathscr{D}$ is the "hourglass-shaped" region, $\mathscr{D}=$ $\left\{z \in \mathbb{C}:\left|\arg \left[z+\left(1+z^{2}\right)^{1 / 2}\right]\right|<d\right\}$, take $\phi(z)=\log \left[z+\left(1+z^{2}\right)^{1 / 2}\right]$. The relation (4.2) reduces to

$$
f(z)=g(z)-\left[g(-\infty)+\left(z+1\left(1+z^{2}\right)^{1 / 2}\right) g(\infty)\right] /\left[1+z+\left(1+z^{2}\right)^{1 / 2}\right],
$$

and the class $\mathbf{L}_{\alpha, \beta}(\mathscr{D})$ is the same as the class of all functions $f \in \operatorname{Hol}(\mathscr{D})$ such that, if $z \in \mathscr{D}$ and $\mathfrak{R} z \leq 0$, then $|f(z)| \leq c(1+|z|)^{-\alpha}$, while if $z \in \mathscr{D}$ and $\mathfrak{R} z \geq 0$, then $|f(z)| \leq c(1+|z|)^{-\beta}$. This map thus allows for algebraic decay at both $x=-\infty$ and $x=\infty$. The Sinc points $z_{j}$ are defined by $z_{j}=$ $\sinh (j h)$, and $1 / \phi^{\prime}\left(z_{j}\right)=\cosh (j h)$. A possible integral to be approximated via this transformation is

$$
p(x)=\int_{-\infty}^{x}(x-t)^{a^{\prime}-1}\left(2+t+t^{2}\right)^{-b^{\prime}} d t .
$$


Example 4.8. If $(a, b)=\mathbb{R}$, and if $\mathscr{D}$ is the "funnel-shaped" region, $\mathscr{D}=\{z \in$ $\left.\mathbb{C}:\left|\arg \left\{\sinh \left[z+\left(1+z^{2}\right)^{1 / 2}\right]\right\}\right|<d\right\}$, take $\phi(z)=\log \left\{\sinh \left[z+\left(1+z^{2}\right)^{1 / 2}\right]\right\}$. The relation (4.2) then reduces to

$f(z)=g(z)-\left[g(-\infty)+\sinh \left(z+\left(1+z^{2}\right)^{1 / 2}\right) g(\infty)\right] /\left[1+\sinh \left(z+\left(1+z^{2}\right)^{1 / 2}\right)\right]$, and the class $\mathbf{L}_{\alpha, \beta}(\mathscr{D})$ is the same as the class of all functions $f \in \operatorname{Hol}(\mathscr{D})$ such that, if $z \in \mathscr{D}$ and $\mathfrak{R} z \leq 0$, then $|f(z)| \leq c(1+|z|)^{-\alpha}$, while if $z \in \mathscr{D}$ and $\mathfrak{R} z \geq 0$, then $|f(z)| \leq c e^{-\beta|z|}$. This map thus allows for algebraic decay at $x=-\infty$ and exponential decay at $x=\infty$. The Sinc points $z_{j}$ are defined by $z_{j}=(1 / 2)\left[t_{j}-1 / t_{j}\right]$, where $t_{j}=\log \left[e^{j h}+\left(1+e^{2 j h}\right)^{1 / 2}\right]$, and $1 / \phi^{\prime}\left(z_{j}\right)=$ $(1 / 2)\left(1+1 / t_{j}^{2}\right)\left(1+e^{-2 j h}\right)^{-1 / 2}$. A possible integral which could be considered via this transformation is

$$
p(x)=\int_{-\infty}^{x}(x-t)^{a^{\prime}-1}\left(1+e^{b^{\prime} t}\right)^{-1.4} d t .
$$

In the above examples, the specific convolution integrals given can be used to identify the constants $\alpha$ and $\beta$ in the space $\mathbf{M}_{\alpha, \beta}(\mathscr{D})$ containing the convolution $p$ or $q$ defined in (1.2). Given a particular convolution integral, it may be possible to bound the integrand in this integral by the integrand in one of the above specific examples. Known identities or asymptotic expressions of hypergeometric or confluent hypergeometric functions can then be used to determine the asymptotic behavior of $p$ or $q$, as $x$ approaches an endpoint, $a$ or $b$, of the interval $(a, b)$. To this end, known limiting relations about the asymptotic forms of $f$ and its classical Laplace transform $\hat{f}$ are frequently also useful.

The second part of the following theorem was stated in [14], and then proved in $[4,5]$ (see also [13]); the first part of the theorem follows readily from the second part.

Theorem 4.9. Let $g \in \mathbf{X}$, and let $h=\gamma / N^{1 / 2}$, for some fixed positive constant $\gamma$. Let $\mathscr{I}$ be defined as in (3.1), and let $\mathscr{I}_{m}$ be defined as in (3.16). Then, as $N \rightarrow \infty$,

$$
\left\|\mathscr{I} g-\mathscr{I}_{m} g\right\|_{\mathbf{Y}} \rightarrow 0 .
$$

In particular, if $g / \phi^{\prime} \in \mathbf{L}_{\alpha, \beta}(\mathscr{D})$, and if $h=[\pi d /(\beta N)]^{1 / 2}$, then there exists a positive constant $c_{1}$ which is independent of $N$ such that

$$
\left\|\mathscr{F} g-\mathscr{I}_{m} g\right\|_{\mathbf{Y}} \leq c_{1} N^{1 / 2} e^{-(\pi d \beta N)^{1 / 2}}
$$

One finds, in the derivation of (4.14), that the constant $c_{1}$ is proportional to $\int_{\partial \mathscr{D}}|g(t) d t|$.

Next, we shall obtain a bound on the difference $F(\mathscr{I}) g-F\left(\mathscr{I}_{m}\right) g$. The main difficulty of carrying out this task results from the fact that $\mathscr{I}$ and $\mathscr{I}_{m}$ do not commute.

At the outset, we establish the convergence of $F\left(\mathscr{J}_{m}\right) g$ to $F(\mathcal{F}) g$, as well as the continuity of the Fréchet derivative of $F$. To establish our notation, we set

$$
\Delta=\mathscr{I}-\mathscr{I}_{m}, \quad \mathscr{I}_{t}=\mathscr{I}_{m}+t \Delta .
$$

Since $\mathscr{I}_{t}=t \mathscr{I}+(1-t) \mathscr{I}_{m}$, and since the spectra of both $\mathscr{I}$ and $\mathscr{I}_{m}$ lie in the closure of $\Omega^{+}$, the same is true of $\mathscr{I}_{t}$, for all $t \in[0,1]$. 
We may now deduce, by proceeding as for (3.11), that

$$
F\left[\mathscr{I}_{t}\right] g=-\frac{1}{2 \pi i} \int_{-i \infty}^{i \infty} \frac{\mathscr{I}_{t}}{s}\left[s-\mathscr{I}_{t}\right]^{-1} F(s) d s g .
$$

Using (4.16), we readily derive the expression

$$
\frac{d}{d t} F\left[\mathscr{I}_{t}\right] g=-\frac{1}{2 \pi i} \int_{-i \infty}^{i \infty}\left[s-\mathscr{I}_{t}\right]^{-1} \Delta\left[s-\mathscr{I}_{t}\right]^{-1} F(s) d s g .
$$

We then find that

$$
\begin{aligned}
& {\left[F(\mathscr{I})-F\left(\mathscr{I}_{m}\right)\right] g=\int_{0}^{1} \frac{d}{d t} F\left[\mathscr{J}_{t}\right] d t g} \\
& \quad=-\frac{1}{2 \pi i} \int_{-i \infty}^{i \infty} \int_{0}^{1}\left[s-\mathscr{J}_{t}\right]^{-1} \Delta\left[s-\mathscr{J}_{t}\right]^{-1} d t F(s) d s g \\
& \quad=-\frac{1}{2 \pi i} \int_{-i \infty}^{i \infty}\left[s-\mathscr{I}_{m}\right]^{-1} \Delta\left[s-\mathscr{I}^{-1} F(s) d s g .\right.
\end{aligned}
$$

These integral expressions will be useful for estimating a norm bound on $\left[F(\mathscr{J})-F\left(\mathscr{J}_{m}\right)\right] g$.

Let us now prove

Lemma 4.10. Let $\mathfrak{R} s \leq-c<0$. Then, for $g \in \mathbf{X}$, we have

(a) as $m \rightarrow \infty$,

$$
\varepsilon_{m} \equiv\left\|[s-\mathscr{I}]^{-1} \Delta\left[s-\mathscr{I}_{m}\right]^{-1} g\right\|_{\mathbf{Y}} \rightarrow 0
$$

(b) as $m \rightarrow \infty$, and for any fuxed $t \in[0,1]$,

$$
\|\left\{\left[s-\mathscr{I}^{-1} \Delta[s-\mathscr{I}]^{-1}-\left[s-\mathscr{I}_{t}\right]^{-1} \Delta\left[s-\mathscr{I}_{t}\right]^{-1}\right\} g \|_{\mathbf{Y}}=o\left(\varepsilon_{m}\right),\right.
$$

where $\varepsilon_{m}$ is defined as in (4.19).

Proof. Set

$$
w=\frac{\mathscr{I}}{s}\left[s-\mathscr{I}^{-1} g, \quad w_{t}=\frac{\mathscr{I}_{t}}{s}\left[s-\mathscr{I}_{t}\right]^{-1} g .\right.
$$

The expression for $w$ can be written in explicit form, namely,

$$
w(x)=\frac{1}{s^{2}} \int_{a}^{x} e^{(x-t) / s} g(t) d t .
$$

Taking the difference between $w$ and $w_{t}$ as given in (4.21) yields

$$
s\left[w-w_{t}\right]-\mathscr{I}_{t}\left[w-w_{t}\right]=\left[\mathscr{J}-\mathscr{I}_{t}\right][w+g / s] .
$$

Since the spectra of both $\mathscr{I}$ and $\mathscr{I}_{m}$ lie in the right half-plane, the same is true of $\mathscr{I}_{t}$, and it thus follows that $\left\|\left[s-\mathscr{J}_{t}\right]^{-1}\right\| \leq 1 / c$. A norm bound on $w-w_{t}$ is thus given by

$$
\left\|w-w_{t}\right\|_{\mathbf{Y}} \leq \frac{\left\|\left[\mathscr{I}-\mathscr{F}_{t}\right](w+g / s)\right\|_{\mathbf{Y}}}{c},
$$

where $w$ is defined in (4.21). The first inequality (4.19) now follows, by Theorem 4.9. 
To prove the inequality (4.20), we start with the following identity, which is valid for all $t \in[0,1]$ :

$$
\begin{aligned}
& \left\{[s-\mathscr{I}]^{-1} \Delta[s-\mathscr{I}]^{-1}-\left[s-\mathscr{I}_{t}\right]^{-1} \Delta\left[s-\mathscr{I}_{t}\right]^{-1}\right\} g \\
& \quad=\left[[s-\mathscr{I}]^{-1}-\left[s-\mathscr{I}_{t}\right]^{-1}\right] \Delta\left\{[s-\mathscr{I}]^{-1}-\left[s-\mathscr{I}_{t}\right]^{-1}\right\} g .
\end{aligned}
$$

Now, if $w$ and $w_{t}$ are defined as in (4.21), we have

$$
w-w_{t}=\left\{\left[s-\mathscr{I}^{-1}-\left[s-\mathscr{I}_{t}\right]^{-1}\right\} g,\right.
$$

and moreover, since by assumption we have $\Re s \leq-c<0$, and since the spectrum of $\mathscr{I}_{t}$ lies on $\Omega^{+}$for all $t \in[0,1]$, we have

$$
\left\|[s-\mathscr{I}]^{-1}\right\| \leq \frac{1}{c}, \quad\left\|\left[s-\dot{\mathscr{F}}_{t}\right]^{-1}\right\| \leq \frac{1}{c} .
$$

The result (4.20) now follows from (4.19), (4.26), and (4.27).

The following result follows directly, from Lemma 4.10, via, e.g., the approximation of $F(s)$ by $F\left(s+2 c_{m}\right)$, with $c_{m}$ playing the role of $c$ in (4.27), and then selecting a sequence $\left\{c_{m}\right\}$ of positive numbers such that $c_{m}$ converges monotonically to 0 as $m \rightarrow \infty$, and such that the right-hand side of (4.24) with $c$ replaced by $c_{m}$ also approaches 0 as $m \rightarrow \infty$.

Lemma 4.10 enables us to come to the following conclusion.

Corollary 4.11. Let the conditions of Lemma 4.10 be satisfied, and for a given $g \in \mathbf{X}$, let $F(\mathscr{I}) g \in \mathbf{Y}$. Then,

(a) as $m \rightarrow \infty$,

$$
\left\|\left\{F(\mathscr{I})-F\left(\mathscr{I}_{m}\right)\right\} g\right\|_{\mathbf{Y}} \rightarrow 0 \text {; }
$$

(b) there exists a constant $c_{1}$ which is independent of $N$ such that

$$
\left\|\left\{F(\mathscr{J})-F\left(\mathscr{J}_{m}\right)\right\} g\right\|_{\mathbf{Y}} \leq c_{1}\left\|\left[\frac{d}{d t} F\left[\mathscr{J}_{t}\right] g\right]_{t=1}\right\|_{\mathbf{Y}} .
$$

Corollary 4.11 establishes the convergence of $F\left(\mathscr{J}_{m}\right) g$ to $F(\mathscr{I}) g$, and it also provides a mechanism for obtaining a bound on the norm $\left\|\left\{F(\mathscr{I})-F\left(\mathscr{I}_{m}\right)\right\} g\right\|_{\mathbf{Y}}$. Assumption 4.12 below enables us to prove the rapid convergence of $F\left(\mathscr{I}_{m}\right) g$ to $F(\mathscr{I}) g$. These assumptions are somewhat more stringent than those required to define the approximation $F\left(\mathscr{J}_{m}\right) g$.

Towards clarifying our notation, Definition 4.1 will continue to be in force, and in particular we let $\mathscr{D}$ and $\mathscr{D}^{\prime}$ be related as in Definition 4.1. In order to introduce properties of the function $f$, we assume the existence of a function $\phi_{f}$ which maps the interval $[0, c]$ onto $\mathbb{R}$, where $c \geq 2(b-a)$, and which conformally maps the domain $\mathscr{D}_{f}$ onto $\mathscr{D}_{d}$. We will also use the notation $\rho_{f}=e^{\phi_{f}}$.

Assumption 4.12. Let the "Laplace transform" of $f$ defined as in (2.3) with $c \geq 2(b-a)$ exist for all $s$ in the right half-plane $\Omega^{+}$, and let $F(s)=\mathscr{O}(s)$ as $s \rightarrow \infty$ in $\Omega^{+}$. Let $g \in \mathbf{X}$, and let $\alpha, \beta, \alpha_{f}$, and $\beta_{f}$ be positive constants such that $0<\alpha \leq 1$, and $0<\beta \leq 1$.

(p) Set

$$
P(r, \tau)=\int_{a}^{\tau} f(r+\tau-\eta) g(\eta) d \eta,
$$


and assume that $P(r, \cdot) \in \mathbf{M}_{\alpha, \beta}\left(\mathscr{D}^{\prime}\right)$, uniformly for $r \in[0, b-a]$, and also, that $\left|P_{r}(r, \tau)\right| \leq c_{1}\left[\rho_{f}(r)\right]^{\alpha_{f}} \phi_{f}^{\prime}(r) /\left[1+\rho_{f}(r)\right]^{\alpha_{f}+\beta_{f}}$, for all $r \in[0, b-a]$ and for all $\tau \in \mathscr{D}$, with $c_{1}$ independent of $r$ and $\tau$.

(q) Set

$$
Q(r, \tau)=\int_{\tau}^{b} f(r+\eta-\tau) g(\eta) d \eta,
$$

and assume that $Q(r, \cdot) \in \mathbf{M}_{\alpha, \beta}\left(\mathscr{D}^{\prime}\right)$, uniformly for $r \in[0, b-a]$ and also, that $\left|Q_{r}(r, \tau)\right| \leq c_{1}\left[\rho_{f}(r)\right]^{\alpha_{f}} \phi_{f}^{\prime}(r) /\left[1+\rho_{f}(r)\right]^{\alpha_{f}+\beta_{f}}$, for all $r \in[0, b-a]$ and for all $\tau \in \mathscr{D}$, with $c_{1}$ independent of $r$ and $\tau$.

The upper limit $c \geq 2(b-a)$ is required in Assumption 4.12 since the (p) and (q) parts of the assumption require that $P$ and $Q$ be analytic functions of $x$, uniformly for $r \in[0, b-a]$; if $c$ in (2.3) were taken to be $b-a$, then our function $f$ as reproduced in our proof of Theorem 4.13 below would vanish when the argument of $f$ would be greater than $b-a$, and it would then not be possible for $P$ and $Q$ to be analytic as assumed in the assumption.

The theorem which follows can also be established, via a slightly more lengthy proof, under the alternate, simpler-to-state conditions that $P(r, \cdot)$ (resp., $Q(r, \cdot)) \in \mathbf{M}_{\alpha, \beta}\left(\mathscr{D}^{\prime}\right)$, uniformly for $r \in[0, b-a]$, and also, that $P(\cdot, \tau)$ (resp., $Q(\cdot, \tau))$ be of bounded variation over $[0, b-a]$, uniformly for $\tau \in \mathscr{D}$.

Theorem 4.13. Let $p$ and $q$ be defined as in (1.2), let $N$ be a positive integer, let $M$ and $m$ be selected as in Definition 4.1, let $A_{m}$ and $B_{m}$ be defined as in (2.2), and let $F, V_{m}$, and $\Pi_{m}$ be defined as in (3.13) and (3.16). Let $h=[\pi d /(\beta N)]^{1 / 2}$.

(i) If $P$ satisfies Assumption $4.12(\mathrm{p})$, then there exists a constant $c_{1}$ which is independent of $N$ such that

$$
\left\|p-\Pi_{m} F\left(A_{m}\right) V_{m} g\right\|_{\mathbf{Y}} \leq c_{1} N^{1 / 2} e^{-(\pi d \beta N)^{1 / 2}} .
$$

(ii) If $Q$ satisfies Assumption 4.12 (q), then there exists a constant $c_{1}$ which is independent of $N$ such that

$$
\left\|q-\Pi_{m} F\left(B_{m}\right) V_{m} g\right\|_{\mathbf{Y}} \leq c_{1} N^{1 / 2} e^{-(\pi d \beta N)^{1 / 2}} .
$$

Proof. We only prove the (i) part of the theorem, since the proof of the (ii) part is almost exactly the same.

We shall use (4.17) and (4.29) in Corollary 4.11.

Replacing $s$ by $1 / s$ in (4.17), and recalling that $F(s)=\hat{f}(1 / s)$, we get

$$
E_{p} \equiv\left[\frac{d}{d t} F\left[\mathscr{I}_{t}\right] g\right]_{t=1}=\frac{1}{2 \pi i} \int_{-i \infty}^{i \infty}(1-s \mathscr{J})^{-1} \Delta(1-s \mathcal{J})^{-1} \hat{f}(s) d s g
$$

with $\Delta$ defined as in (4.15). Now it readily follows that if $g \in \mathbf{X}$, then for any $s \notin \Omega^{+}, s \neq 0$, we have

$$
\left[(1-s \mathcal{I})^{-1} \hat{f}(s) g\right](\tau)=\frac{\partial}{\partial \tau}\left\{\int_{a}^{\tau} e^{s(\tau-\eta)} \hat{f}(s) g(\eta) d \eta\right\},
$$

and applying this result once more, we get

$$
\begin{aligned}
& {\left[(1-s \mathscr{I})^{-1} \Delta(1-s \mathscr{I})^{-1} \hat{f}(s) g\right](x)} \\
& \quad=\frac{\partial}{\partial x} \int_{a}^{x} e^{s(x-t)} \Delta_{\tau}\left\{\frac{\partial}{\partial \tau} \int_{a}^{\tau} e^{s(\tau-\eta)} \hat{f}(s) g(\eta) d \eta\right\}(t) d t,
\end{aligned}
$$


where the subscript $\tau$ on $\Delta$ indicates that $\Delta$ operates with respect to the variable $\tau$. We now apply the operation $(2 \pi i)^{-1} \int_{-i \infty}^{i \infty}[\cdots] d s$ to each side of (4.36) to get

$$
E_{p}(x)=\frac{\partial}{\partial x} \int_{a}^{x} \Delta_{\tau}\left\{\frac{\partial}{\partial \tau} \int_{a}^{\tau} f(x-t+\tau-\eta) g(\eta) d \eta\right\}(t) d t .
$$

(Towards proving the (ii) part of Theorem 4.13, we remark at this point that, if $(\mathscr{I} g)(x)=\int_{x}^{b} g(t) d t$, and if $\mathscr{I}_{m}=\Pi_{m} B_{m} V_{m}$, with $\Pi_{m}, B_{m}$, and $V_{m}$ defined as in (2.4) and (3.16), then the result for $q$ corresponding to the result (4.37) for $p$ is

$$
E_{q}(x)=\frac{\partial}{\partial x} \int_{x}^{b} \Delta_{\tau}\left\{\frac{\partial}{\partial \tau} \int_{\tau}^{b} f(t-x+\eta-\tau) g(\eta) d \eta\right\}(t) d t,
$$

where $\Delta_{\tau}=\mathscr{I}-\mathscr{I}_{m}$ operates with respect to the variable $\tau$.) Let us write

$$
E_{p}=E_{p}^{(1)}+E_{p}^{(2)},
$$

where

$$
\begin{aligned}
& E_{p}^{(1)}(x)=\Delta_{\tau}\left\{\frac{\partial}{\partial \tau} \int_{a}^{\tau} f(\tau-v) g(v) d v\right\}(x)=\Delta_{\tau}\left\{P_{\tau}(0, \cdot)\right\}(x), \\
& E_{p}^{(2)}(x)=\int_{a}^{x} \Delta_{\tau}\left\{P_{x \tau}(x-t, \cdot)\right\}(t) d t .
\end{aligned}
$$

By assumption, $P(r, \cdot) \in \mathbf{M}_{\alpha, \beta}\left(\mathscr{D}^{\prime}\right)$ uniformly with respect to $r \in[0, b-a]$, and it therefore follows that $P_{\tau}(r, \cdot) / \phi^{\prime} \in \mathbf{L}_{\alpha, \beta}(\mathscr{D})$, uniformly for $r \in[0, b-$ a], where $\mathscr{D}$ is defined in terms of $\mathscr{D}^{\prime}$ as in Definition 4.1. Hence it follows immediately, by Theorem 4.9, that there exists a constant $c_{1}$, independent of $N$, such that

$$
\left\|E_{p}^{(1)}\right\|_{\mathbf{Y}} \leq c_{1} N^{1 / 2} e^{-(\pi d \beta N)^{1 / 2}} .
$$

We next bound $E_{p}^{(2)}(x)$. In essence, it will suffice to show that for all $r \in$ $[0, b-a]$ and $\tau \in \mathscr{D}$, we would have the inequality

$$
\left|P_{r \tau}(r, \tau)\right| \leq c_{2} \frac{\left[\rho_{f}(r)\right]^{\alpha} \phi_{f}^{\prime}(r)}{\left[1+\rho_{f}(r)\right]^{\alpha_{f}+\beta_{f}}} \frac{|\rho(\tau)|^{\alpha} \phi^{\prime}(\tau)}{[1+|\rho(\tau)|]^{\alpha+\beta}},
$$

with $c_{2}$ a constant independent of $\tau$ and $r$. For, if (4.42) were to hold, then by Theorem 4.9 we would get

$$
\left|\left\{\Delta_{\tau} P_{r \tau}(r, \tau)\right\}(t)\right| \leq c_{3} \frac{\left[\rho_{f}(r)\right]^{\alpha_{f}} \phi_{f}^{\prime}(r)}{\left[1+\rho_{f}(r)\right]^{\alpha_{f}+\beta_{f}}} N^{1 / 2} e^{-(\pi d \beta N)^{1 / 2}},
$$

with $c_{3}$ a constant independent of $r, \tau$, and $N$. It would then follow that

$$
\begin{aligned}
\left|E_{p}^{(2)}(x)\right| & =\left|\int_{a}^{x}\left\{\Delta_{\tau} P_{x \tau}(x-t, \tau)\right\}(t) d t\right| \\
& \leq c_{3} \int_{a}^{x} \frac{\left[\rho_{f}(x-t)\right]^{\alpha_{f}}}{\left[1+\rho_{f}(x-t)\right]^{\alpha_{f}+\beta_{f}}} \phi_{f}^{\prime}(x-t) d t N^{1 / 2} e^{-(\pi d \beta N)^{1 / 2}} \\
& \leq c_{3} \int_{0}^{c} \frac{\left[\rho_{f}(r)\right]^{\alpha_{f}}}{\left[1+\rho_{f}(r)\right]^{\alpha_{f}+\beta_{f}}} \phi_{f}^{\prime}(r) d r N^{1 / 2} e^{-(\pi d \beta N)^{1 / 2}} \\
& =c_{3} \frac{\Gamma\left(\alpha_{f}\right) \Gamma\left(\beta_{f}\right)}{\Gamma\left(\alpha_{f}+\beta_{f}\right)} N^{1 / 2} e^{-(\pi d \beta N)^{1 / 2}},
\end{aligned}
$$


and we would then get (4.32) by combining (4.39), (4.41), and (4.44).

It remains to derive (4.42). Since $P(r, \cdot) \in \mathbf{M}_{\alpha, \beta}\left(\mathscr{D}^{\prime}\right)$ uniformly with respect to $r \in[0, b-a]$, it follows that $P_{\tau}(r, \cdot) / \phi^{\prime} \in \mathbf{L}_{\alpha, \beta}(\mathscr{D})$ uniformly with respect to $r \in[0, b-a]$. Since for $r \in[0, b-a]$, we also have $\left|P_{r}(\cdot, \tau) / \phi_{f}^{\prime}\right| \leq$ $c_{1}\left[\rho_{f}\right]^{\alpha_{f}} /\left[1+\rho_{f}\right]^{\alpha_{f}+\beta_{f}}$, uniformly for $\tau \in \mathscr{D}$, with $c_{1}$ a constant independent of $\tau$ and $r$, the result (4.42) follows.

\section{Applications}

In this section we illustrate some of the many applications made possible by the discretization of convolution procedure of this paper.

Example 5.1: Laplace transform inversion. Assume that $f$ satisfies the property $f(0)=0$, so that $f(x)=\int_{0}^{x} f^{\prime}(t) d t=\int_{0}^{x} f^{\prime}(x-t) \cdot 1 d t$; i.e., we have a convolution of the form (1.2), in which $g(t)=1$. The Laplace transform equivalent of this last equation in $f$ is $\hat{f}(s)=\{s \hat{f}(s)\}\{1 / s\}$, or, upon replacing $s$ by $1 / s, \hat{f}(1 / s)$ by $F(s)$, and setting $G(s)=F(s) / s$, then in the notation of (3.13), we get

$$
f(x)=(G(\mathscr{I}) 1)(x) .
$$

Using (3.13), (3.17) and (3.18), we get the accurate approximation

$$
f(x) \approx\left(\Pi_{m} G\left(A_{m}\right) \mathbf{1}\right)(x),
$$

where $\mathbf{1}=(1,1, \ldots, 1)^{T}$.

Example 5.2: Feedback control. By classical feedback control theory, the diagram

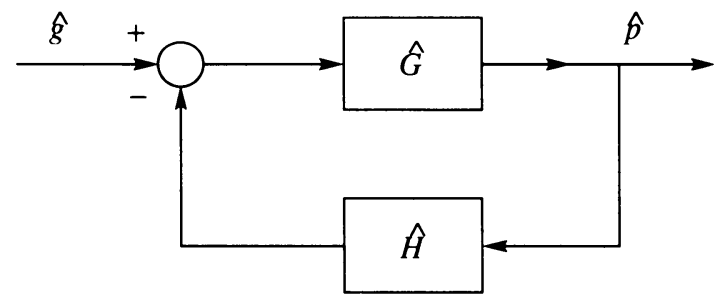

implies the relation

$$
\hat{p}(s)=\frac{\widehat{G}(s)}{1+\widehat{H}(s) \hat{G}(s)} \hat{g}(s),
$$

where the "hat" functions denote standard Laplace transforms, and where a frequently occurring situation in practice is that $g$ as well as the "hat" functions $\widehat{G}$ and $\widehat{H}$ are known, and $p$ is to be determined as a function of time $t$. Upon setting

$$
K(s)=\frac{\widehat{G}(1 / s)}{1+\widehat{H}(1 / s) \widehat{G}(1 / s)},
$$

the method of this paper immediately yields the approximation

$$
p(t) \approx\left(\Pi_{m} K\left(A_{m}\right) V_{m} g\right)(t) .
$$


Example 5.3: Convolution-type integral equations. The method of this paper provides a straightforward method of obtaining accurate solutions to convolution-type integral equations. For example, the Abel integral equation problem

$$
p(t)-\int_{0}^{t}(t-\tau)^{-1 / 3} p(\tau) d \tau=g(t), \quad t \in(0, \infty),
$$

can be dealt with, straightforwardly, either by taking the Laplace transform of each term, solving for $\hat{p}$, and then proceeding as in the previous example, or, noting that the function $f(t) \equiv t^{-1 / 3}$ has the Laplace transform $\hat{f}(s)=$ $\Gamma(2 / 3) / s^{2 / 3}$, by replacing $s$ by $1 / s$ in this last expression to get $F(s)=$ $\Gamma(2 / 3) s^{2 / 3}$, and then solving the system

$$
\left\{I-\Gamma(2 / 3)\left(A_{m}\right)^{2 / 3}\right\} \mathbf{p}=\mathbf{g}
$$

for the vector $\mathbf{p}=\left(p_{-M}, \ldots, p_{N}\right)^{T}$, where $\mathbf{g}=\left(g\left(z_{-M}\right), \ldots, g\left(z_{N}\right)\right)^{T}$. Since $A_{m}=X_{m} D X_{m}^{-1}$, where $D$ is a diagonal matrix (cf. (2.4)), it follows that $A_{m}^{2 / 3}=$ $X_{m} D^{2 / 3} X_{m}^{-1}$. Consequently, (5.7) is equivalent to

$$
\left\{I-\Gamma(2 / 3) D^{2 / 3}\right\} \mathbf{q}=\mathbf{h},
$$

where $\mathbf{q}=X_{m}^{-1} \mathbf{p}$ and $\mathbf{h}=X_{m}^{-1} \mathbf{g}$. The solution $\mathbf{p}$ of (5.7) can therefore be obtained in the following way: First solve $X_{m} \mathbf{h}=\mathbf{g}$ for $\mathbf{h}$, then solve the above linear system for $\mathbf{q}$, and finally compute $\mathbf{p}=X_{m} \mathbf{q}$. We could then use $(2.8)$ to get an approximation of $p$ over $(0, \infty)$. This same procedure could also be used to approximate special functions, expressed as convolution integrals, such as an incomplete Gamma function, a hypergeometric function, or a confluent hypergeometric function. Convolution Fredholm integral equations over a finite interval $(a, b)$, over a semi-infinite interval $(a, b)$, or over all of $\mathbb{R}$ can similarly be dealt with, by using two convolution approximations, one over $(a, x)$ and the other over $(x, b)$. Several numerical tests of this type have already been carried out, and the procedure has been found to be effective.

Example 5.4: Sinc approximation of a three-dimensional convolution. We illustrate here an explicit procedure for approximating a three-dimensional convolution integral of the form

$p\left(x_{1}, x_{2}, x_{3}\right)=\int_{a_{3}}^{x_{3}} \int_{x_{2}}^{b_{2}} \int_{a_{1}}^{x_{1}} f\left(x_{1}-\xi_{1}, \xi_{2}-x_{2}, x_{3}-\xi_{3}\right) g\left(\xi_{1}, \xi_{2}, \xi_{3}\right) d \xi_{1} d \xi_{2} d \xi_{3}$,

where the approximation is sought over the region $\mathbf{B}=\prod_{i=1}^{3} \otimes\left(a_{i}, b_{i}\right)$, and with $\left(a_{i}, b_{i}\right) \subseteq \mathbb{R}$. The reader is instructed to pay particular attention to the method of separation of variables made possible by the one-dimensional convolution approximation, as well as to how well the final algorithm can be adapted to parallel computation. In order to guarantee some accuracy in the final approximation, we shall simply assume, without going into detail, that the function $p$ belongs to the class $\mathbf{M}_{\alpha_{j}, \beta_{j}}\left(\mathscr{D}_{j}^{\prime}\right)$ with respect to each variable $x_{j}$, for all fixed values of the other variables, each in its respective interval of definition, $j=1,2,3$. We shall also assume that the mappings $\phi_{j}: \mathscr{D}_{j}^{\prime} \rightarrow \mathscr{D}_{d^{\prime}}$ have been determined. We furthermore assume that positive integers $N_{j}$ and $M_{j}$ as well as positive numbers $h_{j}(j=1,2,3)$ have been selected such 
that $\left[N_{1} \beta_{1}\right]=\left[N_{2} \beta_{2}\right]=\left[N_{3} \beta_{3}\right]$, such that $M_{j}=\left[\beta_{j} N_{j} / \alpha_{j}\right]$, where [-] denotes the greatest integer function, and such that $h_{j}=\left\{\pi d /\left(\beta_{j} N_{j}\right)\right\}^{1 / 2}$. These definitions ensure that we get the same order of accuracy of approximation $\varepsilon=\mathscr{O}\left(N_{1}^{1 / 2} e^{-\left(\pi d \beta_{1} N_{1}\right)^{1 / 2}}\right)$ in each variable. We set $m_{j}=M_{j}+N_{j}+1$, and we define the Sinc points by $z_{l}^{(j)}=\phi^{-1}\left(l h_{j}\right)$, for $l=-M_{j}, \ldots, N_{j}, j=1,2,3$. Next, we determine matrices $A_{j}, X_{j}$, and $S_{j}$ such that

$$
\begin{aligned}
& A_{j}=h_{j} I_{m_{j}}^{(-1)} D\left(1 / \phi_{j}^{\prime}\right)=X_{j} S_{j} X_{j}^{-1}, \quad j=1,3, \\
& A_{2}=h_{2}\left(I_{m_{2}}^{(-1)}\right)^{T} D\left(1 / \phi_{2}^{\prime}\right)=X_{2} S_{2} X_{2}^{-1} .
\end{aligned}
$$

In (5.9), $I_{m_{j}}^{(-1)}$ is defined in the manner similar to that $I^{(-1)}$ was defined, and the $S_{j}$ are diagonal matrices,

$$
S_{j}=\operatorname{diag}\left[s_{-M_{j}}^{(j)}, \ldots, s_{N_{j}}^{(j)}\right] .
$$

Arbitrarily taking $c_{j} \in\left[2\left(b_{j}-a_{j}\right), \infty\right]$, we set

$$
\begin{aligned}
F\left(s^{(1)}, y, z\right) & =\int_{0}^{c_{1}} f(x, y, z) e^{-x / s^{(1)}} d x, \\
G\left(s^{(1)}, s^{(2)}, z\right) & =\int_{0}^{c_{2}} F\left(s^{(1)}, y, z\right) e^{-y / s^{(2)}} d y, \\
H\left(s^{(1)}, s^{(2)}, s^{(3)}\right) & =\int_{0}^{c_{3}} G\left(s^{(1)}, s^{(2)}, z\right) e^{-z / s^{(3)}} d z .
\end{aligned}
$$

We mention at this point that the functions $F$ and $G$ defined in (5.11) are introduced here solely for purposes of understanding the method of separation of variables described below. Only the function $H$ defined in the last line of (5.11) is required in the final algorithm.

We now illustrate the method of separation of variables. To this end, we first rewrite (5.8) in the notationally more convenient form

$$
p(x, y, z)=\int_{a_{3}}^{z} \int_{y}^{b_{2}} \int_{a_{1}}^{x} f(x-\xi, \eta-y, z-\zeta) g(\xi, \eta, \zeta) d \xi d \eta d \zeta
$$

Discretization with respect to $x$. We set

$$
\mathbf{g}(\eta, \zeta)=\left(g\left(z_{-M_{1}}^{(1)}, \eta, \zeta\right), \ldots, g\left(z_{N_{1}}^{(1)}, \eta, \zeta\right)\right)^{T},
$$

and we then define a vector $\mathbf{p}(y, z)$ by

$$
\mathbf{p}(y, z)=\int_{a_{3}}^{z} \int_{y}^{b_{2}} F\left(A_{1}, \eta-y, z-\zeta\right) \mathbf{g}(\eta, \zeta) d \eta d \zeta,
$$

where $A_{1}$ and $F$ are defined in (5.9) and (5.11), respectively. By the use of the diagonalization identity $A_{1}=X_{1} S_{1} X_{1}^{-1}$ given in (5.9), it now follows from (5.14) that

$$
\mathbf{p}(y, z)=X_{1} \int_{a_{3}}^{z} \int_{y}^{b_{2}}\left(\begin{array}{c}
F\left(s_{-M_{1}}^{(1)}, \eta-y, z-\zeta\right) \\
\ddots \\
F\left(s_{N_{1}}^{(1)}, \eta-y, z-\zeta\right)
\end{array}\right) X_{1}^{-1} \mathbf{g}(\eta, \zeta) d \eta d \zeta .
$$


The expression (5.15) motivates the transformations

$$
\mathbf{h}(\eta, \zeta)=X_{1}^{-1} \mathbf{g}(\eta, \zeta), \quad \mathbf{q}(y, z)=X_{1}^{-1} \mathbf{p}(y, z) .
$$

Thus, if the components of $\mathbf{h}$ and $\mathbf{q}$ are denoted by $h_{i}$ and $q_{i}$, respectively, $i=$ $-M_{1}, \ldots, N_{1}$, equation (5.15) reduces to the decoupled set of scalar equations

$$
q_{i}(y, z)=\int_{a_{3}}^{z} \int_{y}^{b_{2}} F\left(s_{i}^{(1)}, \eta-y, z-\zeta\right) h_{i}(\eta, \zeta) d \eta d \zeta .
$$

Discretization with respect to $y$. We set

$$
\mathbf{h}_{i}(\zeta)=\left(h_{i}\left(z_{-M_{2}}^{(2)}, \zeta\right), \ldots, h_{i}\left(z_{N_{2}}^{(2)}, \zeta\right)\right)^{T},
$$

and we then define a vector $\mathbf{q}_{i}(z)$ by

$$
\mathbf{q}_{i}(z)=\int_{a_{3}}^{z} G\left(s_{i}^{(1)}, A_{2}, z-\zeta\right) \mathbf{h}_{i}(\zeta) d \zeta
$$

where $A_{2}$ and $G$ are defined in (5.9) and (5.11), respectively. By the use of the diagonalization identity $A_{2}=X_{2} S_{2} X_{2}^{-1}$, given in (5.9), it now follows from (5.19) that

$$
\mathbf{q}_{i}(z)=X_{2} \int_{a_{3}}^{z}\left(\begin{array}{c}
G\left(s_{i}^{(1)}, s_{-M_{2}}^{(2)}, z-\zeta\right) \\
\ddots \\
G\left(s_{i}^{(1)}, s_{N_{2}}^{(2)}, z-\zeta\right)
\end{array}\right) X_{2}^{-1} \mathbf{h}_{i}(\zeta) d \zeta .
$$

The last expression motivates the transformations

$$
\mathbf{k}_{i}(\zeta)=X_{2}^{-1} \mathbf{h}_{i}(\zeta), \quad \mathbf{r}_{i}(z)=X_{2}^{-1} \mathbf{q}_{i}(z) \text {. }
$$

If the components of $\mathbf{r}_{i}$ and $\mathbf{k}_{i}$ are denoted by $r_{i, j}$ and $k_{i, j}$, respectively, $i=$ $-M_{1}, \ldots, N_{1}, j=-M_{2}, \ldots, N_{2}$, equation (5.20) reduces to the decoupled set of scalar equations

$$
r_{i, j}(z)=\int_{a_{3}}^{z} G\left(s_{i}^{(1)}, s_{j}^{(2)}, z-\zeta\right) k_{i, j}(\zeta) d \zeta .
$$

Discretization with respect to $z$. We now set

$$
\mathbf{k}_{i, j}=\left(k_{i, j}\left(z_{-M_{3}}^{(3)}\right), \ldots, k_{i, j}\left(z_{N_{3}}^{(3)}\right)\right)^{T},
$$

and we then define a vector $\mathbf{r}_{i, j}$ by

$$
\mathbf{r}_{i, j}=H\left(s_{i}^{(1)}, s_{j}^{(2)}, A_{3}\right) \mathbf{k}_{i, j},
$$

where $A_{3}$ and $H$ are defined in (5.9) and (5.11), respectively. By the use of the diagonalization identity $A_{3}=X_{3} S_{3} X_{3}^{-1}$ given in (5.9), it now follows from (5.24) that

$$
\mathbf{r}_{i, j}=X_{3}\left(\begin{array}{c}
H\left(s_{i}^{(1)}, s_{j}^{(2)}, s_{-M_{3}}^{(3)}\right) \\
\ddots \\
H\left(s_{i}^{(1)}, s_{j}^{(2)}, s_{N_{3}}^{(3)}\right)
\end{array}\right) X_{3}^{-1} \mathbf{k}_{i, j}
$$


The expression (5.25) motivates the transformations

$$
\mathbf{m}_{i, j}=X_{3}^{-1} \mathbf{k}_{i, j}, \quad \mathbf{t}_{i, j}=X_{3}^{-1} \mathbf{r}_{i, j} .
$$

If the components of $\mathbf{m}_{i, j}$ and $\mathbf{t}_{i, j}$ are denoted by $m_{i, j, k}$ and $t_{i, j, k}$, respectively, $i=-M_{1}, \ldots, N_{1}, j=-M_{2}, \ldots, N_{2}, k=-M_{3}, \ldots, N_{3}$, equation (5.25) yields the decoupled set of scalar equations

$$
t_{i, j, k}=H\left(s_{i}^{(1)}, s_{j}^{(2)}, s_{k}^{(3)}\right) m_{i, j, k} .
$$

By assumption, the $m_{i, j, k}$ are known at this point, and (5.27) then determines the $t_{i, j, k}$. The second equation in (5.26) next determines the vectors $\mathbf{r}_{i, j}$. The second equation in (5.21) is then used to determine the vectors $\mathbf{q}_{i}(z)$ at the Sinc points $z_{j}^{(3)}$. The second of $(5.16)$ is next used to determine the vector $\mathbf{p}(y, z)$ at the set of Sinc points $(y, z)=\left(z_{j}^{(2)}, z_{k}^{(3)}\right)$. We can thus recover the complete array of values $p(x, y, z)$ at the set of Sinc points $\left(z_{i}^{(1)}, z_{j}^{(2)}, z_{k}^{(3)}\right)$. The whole procedure is illustrated succinctly via the following algorithm. In this algorithm we use the notation, e.g., $\mathbf{h}_{i, \cdot, k}=\left(h_{i,-M_{2}, k}, \ldots, h_{i, N_{2}, k}\right)^{T}$. We emphasize the obvious ease of adaptation of this algorithm to parallel computation. We also remark that the matrices $X_{j}^{-1}, j=1,2,3$, do not need to be found in the algorithm which follows. For example, in Step 3 of the algorithm, h., $j, k$ satisfies the system of linear equations $X_{1} \mathbf{h}_{, j, k}=\mathbf{g} \cdot, j, k$, and hence one can find the $L U$ factorization of $X_{1}$ and then use it in the back and forward substitutions when solving for all $\mathbf{h}_{\cdot, j, k}$. The same observation applies also to the computation of $\mathbf{k}_{i, \cdot, k}$ in Step 4, and to the computation of $\mathbf{m}_{i, j}, \cdot$ in Step 5 of the algorithm.

\section{Algorithm.}

1. Form the array $z_{i}^{(j)}$, and $\frac{d}{d x} \phi^{(j)}(x)$ at $x=z_{i}^{(j)}$ for $j=1,2,3$, and $i=-M_{j}, \ldots, N_{j}$, and then form the array $\left[g_{i, j, k}\right]=\left[g\left(z_{i}^{(1)}, z_{j}^{(2)}, z_{k}^{(3)}\right)\right]$.

2. Determine $A_{j}, S_{j}$, and $X_{j}$, for $j=1,2,3$, as defined in (5.9). The matrices $X_{j}^{-1}, j=1,2,3$, do not need to be found, as is explained in the above paragraph.

3. Form

$($ See $(5.16))$

$$
\text { h. }, j, k=X_{1}^{-1} \mathbf{g} \cdot, j, k
$$

4. Form

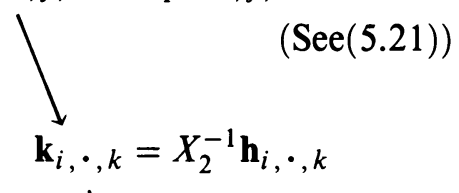

5. Form

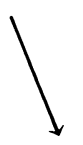

(See (5.26))

$$
\text { p. }, j, k=X_{1} \mathbf{q} \cdot, j, k
$$

[Done]
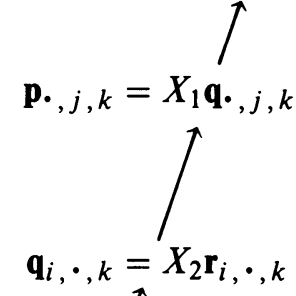

6. Form

$$
\mathbf{m}_{i, j,}=X_{3}^{-1} \mathbf{k}_{i, j} \cdot
$$
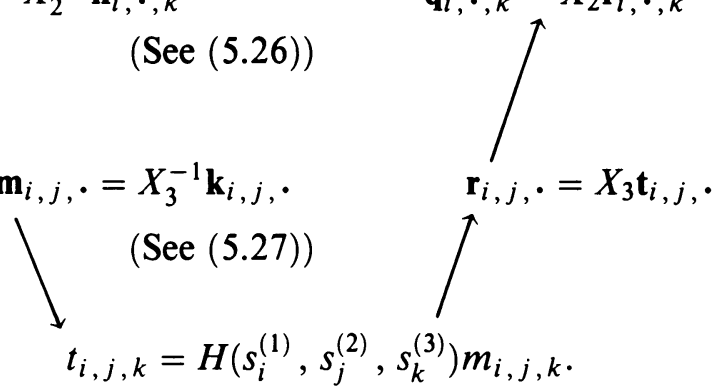
Once the numbers $p_{i, j, k}$ have been computed, we can then use these numbers to approximate $p$ on the region $B$ via the use of a Sinc basis; upon setting $\rho^{(l)}=e^{\phi^{(l)}}$, we can define the functions

$$
\begin{aligned}
\gamma_{i}^{(l)} & =\operatorname{sinc}\left\{\left[\phi^{(l)}-i h\right] / h\right\}, \quad l=1,2,3, i=-M_{l}, \ldots, N_{l}, \\
\omega_{i}^{(l)} & =\gamma_{i}^{(l)}, \quad l=1,2,3, \quad i=-M_{l}+1, \ldots, N_{l}-1,
\end{aligned}
$$

$$
\begin{aligned}
\omega_{-M_{l}}^{(l)} & =\frac{1}{1+\rho^{(l)}}-\sum_{j=-M_{l}+1}^{N_{l}} \frac{1}{1+e^{j h_{l}}} \gamma_{j}^{(l)}, \\
\omega_{N_{l}}^{(l)} & =\frac{\rho^{(l)}}{1+\rho^{(l)}}-\sum_{j=-M_{l}}^{N_{l}-1} \frac{e^{j h_{l}}}{1+e^{j h_{l}}} \gamma_{j}^{(l)} .
\end{aligned}
$$

We then get the approximation

$$
p(x, y, z) \approx \sum_{i=-M_{1}}^{N_{1}} \sum_{j=-M_{2}}^{N_{2}} \sum_{k=-M_{3}}^{N_{3}} p_{i, j, k} \omega_{i}^{(1)}(x) \omega_{j}^{(2)}(y) \omega_{k}^{(3)}(z) .
$$

Assuming a one-dimensional error of the order of $\varepsilon=N_{1}^{1 / 2} e^{-\left(\pi d \beta_{1} N_{1}\right)^{1 / 2}}$, we may expect the approximation in (5.29) to have an error of the order of $\left\{\log \left(N_{1}\right)\right\}^{2} \varepsilon$.

It is also relatively simple to obtain an estimate of the complexity, i.e., the total amount of work required to achieve an error $\varepsilon$ when carrying out the computations of the above algorithm on a sequential machine. By taking $\alpha_{j}=$ $\beta_{j}=\alpha$, and then selecting $M_{j}=N_{j}=N$, it follows that the error in the final approximation is roughly of the order of $\varepsilon=e^{-(\pi d \alpha N)^{1 / 2}}$. The amount of work required to factor the matrices $A_{j}$ into the form $X_{j} S_{j} X_{j}^{-1}$ is of the order of $(2 N+1)^{3}$, and the amount of work in the totality of the matrix-vector multiplications in steps 3 to 6 is of the order of $(2 N+1)^{4}$. Hence the complexity is $\mathscr{O}\left([2 N+1]^{4}\right)=\mathscr{O}\left([\log (\varepsilon)]^{8}\right)$.

Example 5.5: Solving partial differential equations. Solutions of partial differential equations can often be expressed as convolution integrals, or in terms of convolution-type integral equations, via the use of Green's functions. For example, the solution to the problem

$$
\begin{aligned}
u_{t}-u_{x x} & =g(x, t), \quad x \in \mathbb{R}, t>0, \\
u(x, 0) & =0, \quad x \in \mathbb{R},
\end{aligned}
$$

can be expressed in the form

$$
u(x, t)=\int_{0}^{t} \frac{1}{2 \sqrt{\pi(t-\tau)}}\left\{\left(\int_{-\infty}^{x}+\int_{x}^{\infty}\right) e^{-(x-\xi)^{2} / 4(t-\tau)} g(\xi, \tau) d \xi\right\} d \tau
$$

The Green's function in (5.31) is

$$
f(x, t)=\frac{1}{2 \sqrt{\pi t}} e^{-x^{2} / 4 t},
$$

and its "Laplace transform" can be expressed explicitly, i.e.,

$$
\begin{aligned}
F(s, \sigma) & =\int_{0}^{\infty} \int_{0}^{\infty} e^{-x / s-t / \sigma} f(x, t) d x d t \\
& =\frac{s \sigma}{2\left(s+\sigma^{1 / 2}\right)} .
\end{aligned}
$$


We can use the result (5.33) to obtain an explicit procedure for approximating the repeated convolution integral in (5.31). The method is the same as that illustrated for a three-dimensional convolution integral in Example 5.4 above. This procedure has also been tested numerically and found to be effective.

Similarly, the "Laplace transform" of the Green's function for a two-dimensional Poisson problem can be explicitly expressed. The Green's function referred to is

$$
f(x-\xi, y-\eta)=\frac{1}{2 \pi} \log \frac{1}{\sqrt{(x-\xi)^{2}+(y-\eta)^{2}}},
$$

and its "Laplace transform" is

$$
\begin{aligned}
F(s, \sigma) & =\int_{0}^{\infty} \int_{0}^{\infty} e^{-x / s-y / \sigma} f(x, y) d x d y \\
& =\frac{-s^{2} \sigma^{2}}{4\left(s^{2}+\sigma^{2}\right)}-\frac{s \sigma}{2 \pi}\left\{\gamma+\frac{s^{2} \log (s)+\sigma^{2} \log (\sigma)}{s^{2}+\sigma^{2}}\right\},
\end{aligned}
$$

where $\gamma$ denotes Euler's constant.

We remark here that when applying the Sinc discretization procedure of this paper to the approximation of the integral of the Green's function (5.34) times a given function integrated over a rectangular region $(a, b) \times(c, d)$ in the plane, we need to approximate the four convolution integrals, $\int_{a}^{x} \int_{c}^{u}, \int_{a}^{x} \int_{y}^{d}, \int_{x}^{b} \int_{c}^{y}$, and $\int_{x}^{b} \int_{y}^{d}$. Similarly, for three-dimensional potential problems (e.g., when the Green's function is $1 / r$ ) we need to approximate eight integrals. In this latter case it is perhaps worthwhile to mention that it is presently not known whether or not the three-dimensional "Laplace transforms" of convolution kernels such as $1 / r$ or $e^{i k r} / r$ can be explicitly expressed. However, in the final algorithm, the multidimensional "Laplace transform" is required only at discrete points, and the integrals could be evaluated via Sinc quadrature $[13,14,11]$. Since the error of one-dimensional convolution approximation by the method of this paper, using a $(2 N+1) \times(2 N+1)$ matrix, is of the order of $\varepsilon \equiv e^{-c N^{1 / 2}}$, it is readily seen by examination of the algorithm of Example 5.4 that the complexity, i.e., the amount of work required to approximate a $\nu$-dimensional convolution integral via the methods of this paper to within an error $\varepsilon$, is of the order of $[\log (\varepsilon)]^{2 \nu+2}$ in the case when the "Laplace transform" of the convolution kernel is explicitly known. Since the number of points at which the $\nu$-dimensional "Laplace transform" has to be evaluated is of the order of $(2 N+1)^{\nu}$, and since using $N$ points in a one-dimensional Sinc quadrature formula yields an error of the order of $\varepsilon$, the complexity of evaluating the one-dimensional integral at $(2 N+1)^{\nu}$ points via one-dimensional Sinc quadrature is also of the order of $[\log (\varepsilon)]^{2 \nu+2}$. Hence, the order of the complexity to approximate the $\nu$-dimensional convolution by the method of this paper is not increased if the $\nu$-dimensional integral for the "Laplace transform" of the convolution kernel can be reduced explicitly to the approximation of a one-dimensional integral, which can then be evaluated numerically via Sinc quadrature. For example, each of the three-dimensional "Laplace transforms", that of the Poisson kernel, $1 / r$, that of the Helmholtz kernel $e^{i k r} / r$, and that of the heat equation kernel 
$t^{-3 / 2} e^{-r^{2} / t}$, can be explicitly reduced to a one-dimensional integral.

\section{ACKNOWLEDGMENTS}

The author is grateful to the referee for valuable criticisms, and for suggesting the use of the $L U$ factorization of the matrices of eigenvectors $X_{j}$ in the procedure of this paper, and especially in the algorithm in $\S 5$.

\section{BIBLIOGRAPHY}

1. H. Brunner, Discretization of Volterra integral equations of the first kind, Math. Comp. 31 (1977), 708-716.

2. _ A survey of recent advances in the numerical solution of Volterra integral and integro-differential equations, J. Comput. Appl. Math. 8 (1982), 147-163.

3. F. de Hoog and R. Weiss, High order methods for Volterra integral equations of the first kind, SIAM J. Numer. Anal. 10 (1973), 647-664.

4. S. Haber, Two formulas for numerical indefinite integration, Math. Comp. 60 (1993), 279296.

5. R. B. Kearfott, $A$ sinc approximation for the indefinite integral, Math. Comp. 41 (1983), 559-572.

6. P. Linz, A survey of methods for the solution of Volterra integral equations of the first kind, The Application and Numerical Solution of Integral Equations (R. S. Anderssen, Frank R. de Hoog, and Mark Lukas, eds.), Sijthoff \& Noordhoff, Germantown, MD, 1980, pp. 183-194.

7. __ Analytical and numerical methods for Volterra equations, SIAM, Philadelphia, PA, 1985.

8. C. Lubich, Convolution quadrature and discretized operational calculus. I, Numer. Math. 52 (1988), 129-145.

9. $ـ$ Convolution quadrature and discretized operational calculus. II, Numer. Math. $\mathbf{5 2}$ (1988), 413-425.

10. Ch. Lubich and A. Ostermann, Runge-Kutta methods for parabolic equations and convolution quadrature, Math. Comp. 60 (1993), 105-131.

11. J. Lund and K. L. Bowers, Sinc methods for quadrature and differential equations, SIAM, Philadelphia, PA, 1992.

12. B. Riley, $A$ sinc collocation method for weakly singular integral equations, Computation and Control (K. L. Bowers and J. Lund, eds.), Birkhäuser, Basel, 1989, pp. 263-275.

13. F. Stenger, Numerical methods based on sinc and analytic functions, Springer-Verlag, New York, 1993.

14. _ Numerical methods based on Whittaker cardinal, or sinc functions, SIAM Rev. 23 (1981), 165-224.

15. M. Stromberg, Solution of shock problems by methods using sinc functions, Ph.D. thesis, University of Utah, 1988.

16. $\ldots$, Approximate solution of quasilinear equations of conservation law type, Computation and Control (K. L. Bowers and J. Lund, eds.), Birkhäuser, Basel, 1989, pp. 316-331.

17. D. V. Widder, The Laplace transform, Princeton Univ. Press, Princeton, NJ, 1936.

18. A. Young, The application of approximate product integration to the numerical solution of integral equations, Proc. Roy. Soc. London Ser. A 224 (1954), 561-573.

Department of Computer Science, University of Utah, Salt lake City, Utah 84112

E-mail address: stenger@cs .utah.edu 